\title{
Development and application of ANN model for property prediction of supercritical kerosene
}

\author{
Bo Li ${ }^{\mathrm{a}, \mathrm{b}}$, Yachao Lee ${ }^{\mathrm{a}, \mathrm{b}}$, Wei Yao ${ }^{\mathrm{a}, \mathrm{b}, *}$, Yang Lu ${ }^{\mathrm{a}}$, Xuejun Fan ${ }^{\mathrm{a}, \mathrm{b}, *}$ \\ ${ }^{a}$ Key Laboratory of High-Temperature Gas Dynamics, Institute of Mechanics, CAS, Beijing 100190, China \\ ${ }^{\mathrm{b}}$ School of Engineering Science, University of Chinese Academy of Science, Beijing 100049, China
}

\section{A R T I C L E I N F O}

\section{Article history:}

Received 21 December 2019

Revised 6 June 2020

Accepted 2 July 2020

Available online 4 July 2020

\section{Keywords:}

Artificial neural network (ANN)

Principle of Extended Corresponding State (ECS)

RP-3 kerosene

Superitical pressure

OpenFOAM

\begin{abstract}
A B S T R A C T
Three artificial neural network (ANN) models were developed to predict the fluid properties of China RP3 kerosene under supercritical pressure in replacement of the time-consuming property calculations by the principle of Extended Corresponding State (ECS). The analysis shows that the properties predicted by the trained ANN models agree well with the calculations by the ECS method. The correlation coefficients (R) between the ANN predictions and the ECS calculations are higher than 0.99, and most of the relative errors are lower than $0.1 \%$. The prediction by the ANN models is of several orders $\left(10^{4}\right)$ faster than that by the ECS method, especially near the critical points. The trained ANN model was further coupled with the CFD modeling of a realistic kerosene jet, where high efficiency and satisfactory accuracy were shown compared with the direct ECS calculations.
\end{abstract}

(c) 2020 Elsevier Ltd. All rights reserved.

\section{Introduction}

For hypersonic vehicles, passive cooling by using advanced materials is becoming insufficient at Mach number (Ma) of $10+$, where active cooling by using regenerative fuel becomes necessary [1]. Convective heat transfer of hydrocarbon fuels under supercritical pressure has been widely utilized in the regenerative cooling technology [2,3]. The application of regenerative cooling not only effectively cools high-temperature parts but also improves the energy utilization and combustion efficiency through preheating the fuel $[4,5]$. During the regenerative cooling, the fuel not only absorbs heat using its sensible heat sink but also absorbs heat through endothermic chemical reactions using its chemical heat sink [3]. However, abnormal heat transfer usually occurs around the critical point [6,7], as the thermophysical and transport properties of supercritical fluids will change sharply with the temperature and the pressure when approaching the critical point [8]. Supercritical fluid has gas-like transport properties (e.g., viscosity) but large density similar to the liquid [9]. Therefore, accurate prediction of the supercritical properties is essential for thermal management $[10,11]$.

\footnotetext{
* Corresponding authors

E-mail addresses: libo2@imech.ac.cn (B. Li), liyachao@imech.ac.cn (Y. Lee), weiyao@imech.ac.cn (W. Yao), luyang@imech.ac.cn (Y. Lu), xfan@imech.ac.cn (X. Fan).
}

Compared to hydrogen fuels, kerosene is an ideal coolant for its high energy density, small storage volume, and economy [12]. However, most current heat transfer researches focus on pure hydrocarbons, while very few studies focus on the supercritical heat transfer of kerosene [13-15]. On the one hand, this is due to the complex composition of kerosene, which typically contains thousands of compositions, and the specific composition varies with the origin, manufacturer, production year, and some other conditions. On the other hand, this is due to the complex flow and heat transfer phenomena near the critical and vapor-liquid-coexist statuses. For the former, surrogate fuels must be used to simulate the physical and chemical properties of kerosene, while for the latter accurate equation of state must be adopted to solve the thermodynamic properties under supercritical conditions where the real-gas effect must be taken into account [16]. Calibrated by the composition and the heat transfer characteristics of China RP-3 kerosene, several physical surrogate models, such as Fan's three-component model [17], Cheng's five-component model [18] and Zhong's tencomponent model [19], have been proposed to model the regenerative cooling of kerosene. Compared with the cubic equations of state (EoS), such as the Van der Waals equation, Peng-Robinson equation [20] and Redlich-Kwong-Soave equation [21], the principle of Extended Corresponding State (ECS) [6,9,22-25] can provide highly accurate properties across the liquid-vapor-supercritical regimes for a large variety of hydrocarbons. However, the ECS calculation is time-costly for use in three-dimensional (3-D) Computational Fluid Dynamics (CFD) modelings, especially near the crit- 


\begin{tabular}{|c|c|}
\hline \multicolumn{2}{|c|}{ Nomenclature } \\
\hline ANN & Artificial neural network \\
\hline ECS & Extended Corresponding State \\
\hline$T$ & Temperature $(\mathrm{K})$ \\
\hline$P$ & Pressure (MPa) \\
\hline$\rho$ & Density $\left(\mathrm{kg} / \mathrm{m}^{3}\right)$ \\
\hline$a$ & sound speed $(\mathrm{m} / \mathrm{s})$ \\
\hline$\gamma$ & specific heat ratio \\
\hline$P_{r}$ & Prandtl number \\
\hline LES & Large Eddy Simulation \\
\hline SGS & subgrid-scale \\
\hline$k$ & thermal conductivity $(\mathrm{W} / \mathrm{m} \bullet \mathrm{K})$ \\
\hline$\mu$ & Viscosity $(\mathrm{Pa} \bullet \mathrm{s})$ \\
\hline$C_{p}$ & the specific heat at constant pressure $(\mathrm{J} / \mathrm{kg} \bullet \mathrm{K})$ \\
\hline$m$ & the momentum \\
\hline W & the weight in the ANN structure \\
\hline$l_{r}$ & The learning rate \\
\hline
\end{tabular}

ical point $[26,27]$. It is desirable to develop an efficient yet accurate way to evaluate the fluid properties of kerosene, which would avoid the time-consuming, iterative solution and make CFD modeling coupled with real-gas effects more computationally-affordable.

The artificial neural network (ANN), originating from the biological neural network (BNN) [28], is one of the most widely used statistical learning algorithms in recent years and has been successfully applied in function approximation, classification, time series prediction, filtering, data association and optimization $[10,27,29,30]$. The ANN structure functions like biological neurons with the ability to learn from a set of input-output parameter space it is subjected to, and then predicted the output for a new sample set at much high speed and with a sufficient level of accuracy [2]. Usually, the most important advantages of ANN are its high flexibility to simulate nonlinear problems and the capability to approximate any functions with high-dimensional data requiring no detailed information about the system. ANN can handle large and complex interrelated parameters by simply ignoring excess data that are of minimal significance and instead concentrating on the more important inputs [31]. Among several types of ANNs, the backpropagation artificial neural network (BP-ANN) is the most widely applied [32-34]. The main features of BP-ANN are that the weights are automatically adjusted by the information transmitting forward and the errors transferring reversely. However, there is a disadvantage of ANN that it cannot be used to find or explain physical laws involved in a phenomenon because the mathematical information that contributes to the understanding of physical and thermodynamic comportment can only be obtained by solving equations [35]. Nevertheless, ANN is still beneficial in many fields of knowledge. ANN is a feasible method to improve the calculation efficiency of complex nonlinear problems and has been successfully applied to predict the material properties [32,35-40].

Several attempts have been made to alleviate the computationally-expensive situation in properties solving [4146]. Vigor et al. [46] implemented the tabulation-based and correlated dynamic evaluation (CDE) methods to the numerical simulation of supercritical mixing between liquid oxygen and gaseous methane flow around a splitter-plate. Milan et al. [41] extended the above two methods to the simulation of supercritical combustion in their later study. Though multi-dimensional homogeneous tabulation method is more straightforward, faster, and effective compared with the solving of EOS equations, the large table size and the growing memory requirement limit its application in CFD. With the rapid development of deep learning techniques, some researches have successfully replaced the multi-dimensional and memory-intensive tabulation method by efficient neural networks [42-44]. Owoyele et al. [43] investigated the applicability of ANNs to replace the tabulated flamelets and related approaches, whose low memory requirement and low computational cost have been proved by modeling the methyl decanoate combustion in a compression ignition engine. Recently, Milan et al. [42] used the Soave-Redlich-Kwong equation of state and a deep feedforward neural network to estimate the real-fluid thermophysical properties, which is proved to be accurate yet efficient in the LES modeling of supercritical turbulent mixing in a liquid rocket engine.

In this study, a BP-ANN model with three hidden layers will be used to estimate the fluid properties of supercritical kerosene, which includes the equation of state, the thermodynamic and transport properties, and the fluid fraction, in replacement of the computationally-expansive ECS method. A mapping between the thermochemical states and the fluid properties of kerosene will be constructed by ANN to improve the calculation efficiency. The developed ANN structure includes two inputs and seven output parameters. In this study, the pressure $(p)$ and temperature $(T)$ are chosen as the two input quantities, and the output parameters are density $\left(\rho, \mathrm{g} / \mathrm{cm}^{3}\right)$, sound speed $(a, \mathrm{~m} / \mathrm{s})$, viscosity $\left(\mu, P a^{*} \mathrm{~s}\right)$, thermal conductivity $\left(\mathrm{k}, \mathrm{W} / \mathrm{m}^{*} \mathrm{~K}\right)$, specific heat at constant pressure $\left(C_{p}\right.$, $\left.\mathrm{J} / \mathrm{g}^{*} \mathrm{~K}\right)$, specific heat ratio $(\gamma)$ and Prandtl number $\left(P_{r}\right)$. According to the working conditions of the cooling channels of hypersonic vehicles, the training databases were generated by the ECS method with the temperature range from $300 \mathrm{~K}$ to $1000 \mathrm{~K}$, and the pressure range from 2.5 MPa to 6.0 MPa. Three ANN training databases are built respectively for the aforementioned three kerosene surrogate models, i.e., Fan's three-component model [17], Cheng's fivecomponent model [18] and Zhong's ten-component model [19]. The accuracy and efficiency of the developed ANN model will be validated against the exact ECS results. The performance of the developed ANN model will be further analyzed through coupling into the CFD modelings.

\section{ANN model and its training process in OpenFOAM}

\subsection{Algorithm of BP-ANN}

As shown in Fig. 1, a typical multilayer BP-ANN architecture usually consists of three main parts: an input layer, some hidden layers, and an output layer. Each layer is composed of single neurons operating in parallel. The input layer is used to load the known parameters $X$, which directly determine the output results, such as pressure $p$ and temperature $T$ in this study. The hidden layer is set to accommodate all the known information and build a nonlinear relationship between the input parameters and the output results. The output layer is utilized to resolve the unknown results $Y$ from the hidden layer. Once the number of hidden layers and the number of neurons in each hidden layer were determined, the connection weights between the neurons were adjusted by information transmitting forward and errors transferring reversely during the process of training. Any errors made by the network during training get sent backward through it in an attempt to correct it and teach the network what is right and wrong, as schematically illustrated in Figs. 1 and 2. During the learning process, the error is estimated by the mean squared error (MSE) defined as,

$M S E=\frac{1}{n} \sum_{i=1}^{n}\left(y_{i}-t_{i}\right)^{2}$

where $y_{i}, n$ is the network output value and the total number respectively, $t_{i}$ is the target value. Smaller MSE values indicate better ANN performance. When the MSE reaches a minimum, or the steps of training reach the predefined maximum, the network training is considered complete, and the weights are stored. 


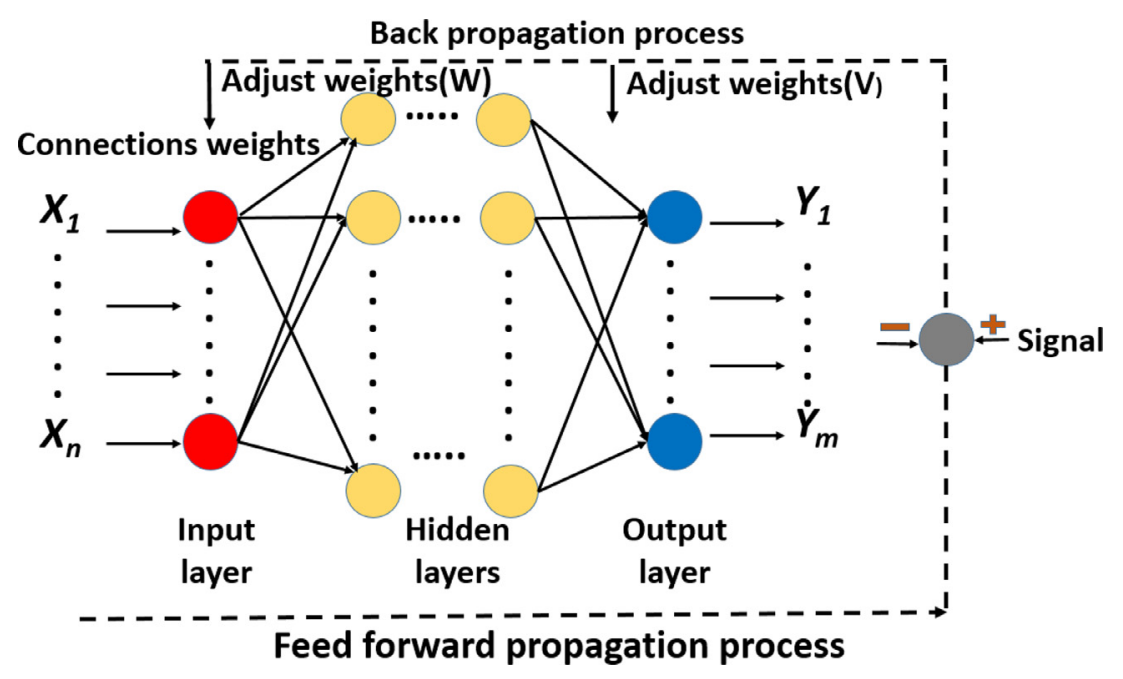

Fig. 1. Schematic diagram of three-layer BP-ANN.

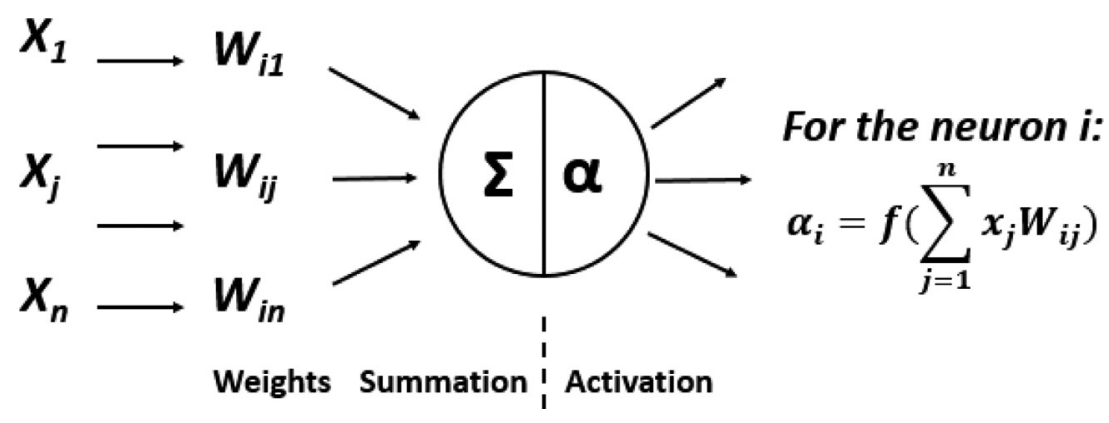

Fig. 2. Information processing in a single neural-network neuron.

Fig. 2 illustrates how signals are processed through a single neuron. First, the neuron receives weighted activations of other neurons through its incoming connections and added up. The summation is then passed through an activation function $f$, the outcome $\alpha$ being the new activation, which is multiplied by the specific weight and transferred to the next layer's neurons. The activation function calculates the output of a deep learning model through a nonlinear transformation on the input. The activation function determines the accuracy and computational efficiency of a trained model. During the process of backpropagation, the gradients are supplied along with the error to update the weights and biases, so the activation functions should be differentiable and monotonic. The logistic sigmoid, hyperbolic tangent (tanh), and rectified linear unit (ReLU) are the standard nonlinear activation functions in deep learning, with the sigmoid function most frequently used. Both the sigmoid and tanh functions have S-Shaped curves. The only difference is that the outputs of the sigmoid function lie between 0 and 1 , while the outputs of the tanh function lie between 1 and -1 . The learning rate using the tanh function will be lower because of the wide-spanning range of 2 compared with 1 in the sigmoid function. The output of the tanh function is zero centered, which makes the optimization easier since the neurons in the later layers of the network receive inputs that are zero-centered. The ReLU function can significantly accelerate the convergence of stochastic gradient descent compared with the sigmoid and tanh activation functions. However, the ReLU function has a vital drawback called "dying neuron", which will stop responding if it is not activated initially. The sigmoid function is differentiable across its entire domain and is easy to compute with a faster convergence rate. Previous studies have shown that when a network has been successfully trained, different activation func- tion has approximately the same effect. In addition to the selection of an activation function, the training algorithms, network sizing, and learning parameters are more vital for the proper training of the network. In this study, a logistic sigmoid activation function is used:

$f(z)=\frac{1}{1+e^{-z_{n}}}$

where $z_{n}=\sum_{j=1}^{n} x_{j} W_{i j}$ is the weighted sum of the input activations for each neuron. The comparison of the training rate using different activation functions merits future study.

During the backpropagation, the weights were updated according to the gradient descent algorithm, which has been detailedly introduced in the literature [47-49]. The change of weight $W_{i j}$ in the connection neuron from $i$ to $j$ can be written as

$\Delta W_{i j}=-l_{r} \frac{\partial M S E}{\partial W_{i j}}$

Here an appropriate learning rate $l_{r}$ plays an important role in the training, since the abrupt change caused by a large $l_{r}$ may miss the minimum while the low learning rate caused by a small $l_{r}$ slows down the training. In order to minimize the error function MSE, $W_{i j}$ needs to be decreased when $\frac{\partial M S E}{\partial W_{i j}}>0$, and increased when $\frac{\partial M S E}{\partial W_{i j}}<0$. In order to avoid oscillation inside the network such as alternating connection weights, and to improve the convergence rate, an adaptive learning rate was adopted [50]. Similar to a ball rolling down a mountain, the current rolling speed is determined not only by the local slope of the mountain but also by its own inertia (momentum). Similarly, the change of weight $W_{i j}$ at time 


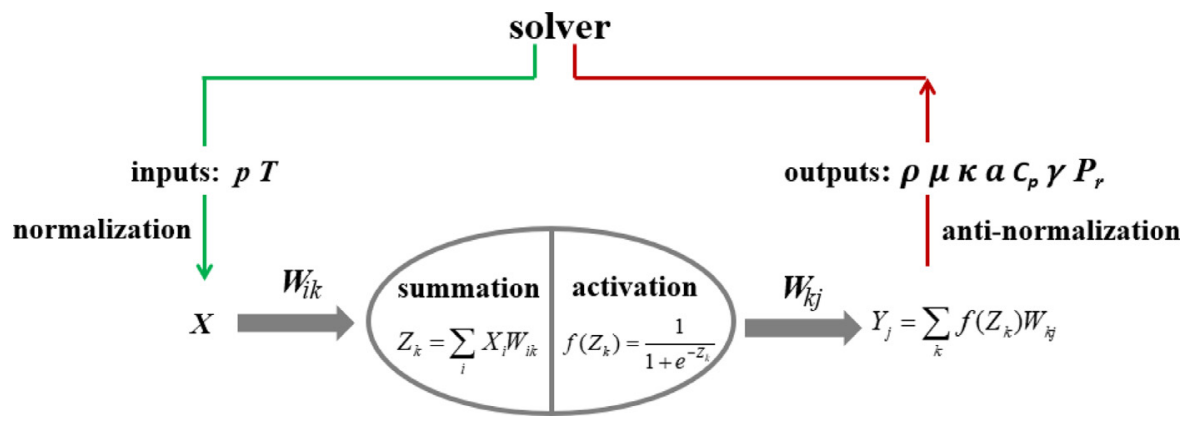

Fig. 3. Invocation process in the BP-ANN library.

$(t+1)$ is $\Delta W_{i j}(t+1)$, written as

$\Delta W_{i j}(t+1)=(\mathrm{m}-1) l_{r} \frac{\partial M S E}{\partial W_{i j}}+m \Delta W_{i j}(t)$

where $m$ is the momentum varying in the range of $[0,1]$. Momentum is not only a technique to keep the weight changing in the direction of the previous step to speed up the convergence but also has the benefit of avoiding the abrupt changing in the opposite direction once the direction reverses. Because the influence of the momentum in the weight update can become too large, a momentum adaptation technique was also developed to use in combination with the learning rate adaptation [51].

\subsection{Coupled BP-ANN into OpenFOAM}

To implement the BP-ANN in the open-source platforms OpenFOAM [3], an ANN library was established, which could be directly invoked in CFD applications. The ANN library has two main functionalities. The first functionality of the library is the training of the weight matrices $W$, which is usually finished before the calculations. The training may be invoked during the calculations only when there is an update on the thermophysical properties. The adaptive learning rate and multiple hidden layers are implemented in the library to accelerate the training. The second functionality is outputting the required results by invoking the trained artificial neural network with trained weight matrices. Such a mechanism works like a "black box", where no direct or explicit relations have ever been established between the input parameters and the output results. The basic process of this procedure is shown in Fig. 3.

\subsection{Databases preparation and training process}

Usually, the state curves of different fluids are quite close when the state variables are dimensionalized by critical parameters [17]. Based on that fact, the Corresponding State (CS) principle [17] predicts unknown properties from the known properties by transformed relations. However, only a small number of fluids strictly follow the ECS principle. The variation of molecule shapes and the resulting dipole moment are the main sources of errors. To remedy this, an acentric factor " $\omega$ " and some other correlation parameters are included in the Extended Corresponding State (ECS) principle. The acentric factor can be written as:

$\omega=-\log \left(\frac{P^{s}}{P^{c}}\right)-1.0$

where $P^{s}$ is the saturated pressure at $T / T_{C}=0.7$. The correlation coefficient can be written as:

$h_{x}=\left(\rho_{r}^{c} / \rho^{c}\right) \varphi\left(T / T_{c}, \rho / \rho_{c}, \omega\right)$

$f_{x}=\left(T^{c} / T_{r}^{c}\right) \theta\left(T / T_{c}, \rho / \rho_{c}, \omega\right)$
Table 1

Training database for each surrogate model of kerosene.

\begin{tabular}{lllll}
\hline Kerosene & $\mathrm{P}(\mathrm{MPa})$ & $\mathrm{T}(\mathrm{K})$ & $\Delta \mathrm{T}(\mathrm{K})$ & Volume \\
\hline $3,5,10$-component & $2.5-6$ & $300-600$ & 5 & 49,086 \\
& & $600-700$ & 0.1 & \\
& & $700-1000$ & 1 &
\end{tabular}

where the subscript $r$ represents the parameters of the reference fluid, the script $c$ represents the critical state. The shape factors $\varphi$ and $\theta$ can be solved by the relationship between the saturated density and the saturated pressure of the two fluids. For multicomponent mixtures, a hypothetical pure fluid can be approximated, and its properties can be obtained from the ECS laws of the corresponding components by certain mixing rules [52,53].

It is well known that a reliable data source is a necessary prerequisite to the successful development of ANN models. This work aims to train three accurate yet efficient ANN models to predict the properties of China RP-3 kerosene under supercritical pressures. Considering the difficulty of obtaining sufficient and reliable experimental data for the supercritical kerosene, all the training data are established from the ECS calculations in this study, with the parameters confined between $2.5 \mathrm{MPa} \leq P \leq 6 \mathrm{MPa}$ and $300 \mathrm{~K} \leq T \leq 1000 \mathrm{~K}$. Insufficient training data source will reduce the accuracy of the prediction by the BP-ANN, while excessive training data source will also slow down the training process of the BPANN. Through trial and error, the most suitable training database for each surrogate model is determined and listed in Table 1 . The increment for pressure $\Delta P$ is $0.1 \mathrm{MPa}$, while the increment for temperature $\Delta T$ varies from $0.1 \mathrm{~K}$ to $5 \mathrm{~K}$ for different temperature ranges. The total number of data points for each training database is 49,086 .

Theoretically, ANN has no restriction on its training data despite the fact that the order of magnitude of the original sample may vary greatly. However, to achieve a higher training accuracy, it's better to put the training data source at the same order before each training process. Therefore, all the input and output variables are normalized to the range of $[0,1]$ by

$X^{\prime}=\frac{X-X_{\min }}{X_{\max }-X_{\min }}$

where $\mathrm{X}$ is the original value, $\mathrm{X}^{\prime}$ is the normalized value, and $\mathrm{X}_{\max }$ and $X_{\min }$ are the maximum and minimum of $X$, respectively. The database is divided by the $\mathrm{K}$ Fold cross-validation method, which can provide sufficient data for the model training as well as validation. In the cross-validation, each training database was equally divided into ten subsets after randomly disrupting the order, and one of the ten subsets was used as the validation set while the other nine subsets were put together to form a training set. Both training and validation errors are monitored. In addition to the training database, an additional data set is generated by the ECS method to 
Table 2

The influence of neurons in each hidden layer and maximum training epochs.

\begin{tabular}{|c|c|c|c|c|}
\hline Kerosene & Neurons & Epochs & TrainingSetMSE & ValidationSetMSE \\
\hline \multirow[t]{5}{*}{ 3-component } & $(30,30,30)$ & 5,000 & $1.255 \mathrm{e}-06$ & $1.260 \mathrm{e}-03$ \\
\hline & $(30,30,30)$ & 10,000 & $3.661 \mathrm{e}-07$ & $1.043 \mathrm{e}-03$ \\
\hline & $(30,30,30)$ & 50,000 & $1.249 \mathrm{e}-07$ & $5.747 e-04$ \\
\hline & $(30,30,30)$ & 100,000 & $7.359 \mathrm{e}-08$ & $4.109 \mathrm{e}-04$ \\
\hline & $(30,30,30)$ & 300,000 & $3.752 \mathrm{e}-08$ & $2.834 \mathrm{e}-04$ \\
\hline 3-component & $(50,50,50)$ & 300,000 & $3.683 e-08$ & $2.601 \mathrm{e}-04$ \\
\hline \multirow[t]{6}{*}{ 5-component $=$} & $(30,30,30)$ & 5,000 & $1.586 \mathrm{e}-05$ & $2.158 \mathrm{e}-03$ \\
\hline & $(30,30,30)$ & 50,000 & $1.851 \mathrm{e}-07$ & $6.784 \mathrm{e}-04$ \\
\hline & $(30,30,30)$ & 100,000 & $1.201 \mathrm{e}-07$ & $5.886 e-04$ \\
\hline & $(30,30,30)$ & 300,000 & $1.163 e-07$ & $5.683 e-04$ \\
\hline & $(50,50,50)$ & 100,000 & $1.086 \mathrm{e}-07$ & $5.735 e-04$ \\
\hline & $(50,50,50)$ & 300,000 & $6.523 e-08$ & $4.984 \mathrm{e}-04$ \\
\hline \multirow[t]{2}{*}{ 10-component } & $(50,50,50)$ & 100,000 & $6.416 e-08$ & $5.060 \mathrm{e}-04$ \\
\hline & $(50,50,50)$ & 300,000 & $3.669 e-08$ & $3.813 e-04$ \\
\hline
\end{tabular}

Table 3

The best ANN structures for each surrogate model of China RP-3 kerosene.

\begin{tabular}{lllll}
\hline Kerosene & Neurons & Epochs & TrainingSetMSE & ValidationSetMSE \\
\hline 3-component & $(30,30,30)$ & 300,000 & $3.752 \mathrm{e}-08$ & $2.834 \mathrm{e}-04$ \\
5-component & $(50,50,50)$ & 300,000 & $6.523 \mathrm{e}-08$ & $4.984 \mathrm{e}-04$ \\
10-component & $(50,50,50)$ & 300,000 & $3.669 \mathrm{e}-08$ & $3.813 \mathrm{e}-04$ \\
\hline
\end{tabular}

assess the overall accuracy of the trained ANN models as well as to compare their performance.

Another important aspect of the development of ANN models is the network structure, including the numbers of neurons of the input and output layers, the number of hidden layers, and the number of neurons in each layer. The numbers of neurons in the input and output layers are fixed values, usually determined by the examined problem. However, the number of hidden layers and the number of neurons in each layer is still an open question for diffident problems. Usually, the more hidden layers and neurons in the ANN model, the better the ANN model can capture the nonlinear relationship between the input and the output, whereas the more difficult and slower to train it. By trial and error, it is found that an ANN model with three hidden layers and 30-50 neurons in each layer can accurately predict the thermophysical properties of kerosene under supercritical pressures without introducing excessive computational burden. After fixing the structure of the ANN model, the results also change obviously with the total training epochs. It is usually observed that training error decreases steadily over time, but the validation set error may begin to rise again, which is called overfitting. To avoid overfitting and make sure that the final trained model performs well not just on the training data but also on new inputs, a regularization strategy of early stopping was adopted in this study. Early stopping is an unobtrusive form of regularization because it requires nearly no change in the underlying training procedure, the objective function, and the set of allowable parameter values. It is also the reason why the MSE equation (Eq. (1)) doesn't contain an explicit regularization term. The early stopping strategy works by monitoring the validation set error to terminate the trajectory at a point with the lowest validation set error. In this view, the number of training steps is just another hy- perparameter. Usually, when the training epochs reach a certain number, the validation set error (ValidationSetMSE) will reach a small value and drops negligibly later. In order to choose the most appropriate number of neurons in each hidden layer and the suitable total training epochs considering both the training efficiency and accuracy, comparisons for each surrogate model of China RP-3 kerosene were shown in Table 2.

Usually, the smaller the mean squared errors (MSEs) of training and validation are, the better result the ANN model can predict. By analyzing the result of the test subset, it is found that the orders of magnitude for the MSE of the training subset (abbreviated as TrainingSetMSE) and the validation subset (abbreviated as validationSetMSE) should be as small as $10^{-8}$ and $10^{-4}$ respectively. As seen from Table 2, both the training and validation MSEs of the (50 5050 ) case for the 3-component kerosene are only slightly smaller than the (30 3030 ) ones, while the training cost of the $(50,50,50)$ case is almost three times that of the $(30,30,30)$ one. Since a more complex ANN structure would result in lower prediction efficiency, the (30 3030 ) case is determined to be the best ANN structure for the 3-component kerosene. However, note that the accuracy of the ANN predictions will always increase with the increasing of neuron numbers in each hidden layer, assuming enough training. As seen from the training results for the 3- and 5-component surrogate models, the ANN model with neurons of (50 50 50) in each hidden layer produces smaller traing and validation errors compared with the $(30,30,30)$ cases, after 300,000 -epoch training. Usually, the more complex surrogate model requires a more complex ANN structure. For the 10-component kerosene surrogate, only the ANN structure with $(50,50,50)$ neurons can produce a satisfatory prediction. The best ANN structures and training epochs for each surrogate model of China RP-3 kerosene are listed in Table 3.

Table 4

The randomly chosen five set samples for each surrogate model of kerosene.

\begin{tabular}{llll}
\hline Kerosene & $\mathrm{P}(\mathrm{MPa})$ & $\mathrm{T}(\mathrm{K})$ & Volume \\
\hline 3-component & $2.55,3.17,3.76,4.84,5.91$ & $300-1000, \Delta \mathrm{T}=1$ & 701 \\
5-component & $2.62,3.26,3.89,4.31,5.87$ & & \\
10-component & $2.53,3.38,3.68,4.72,5.79$ & & \\
\hline
\end{tabular}



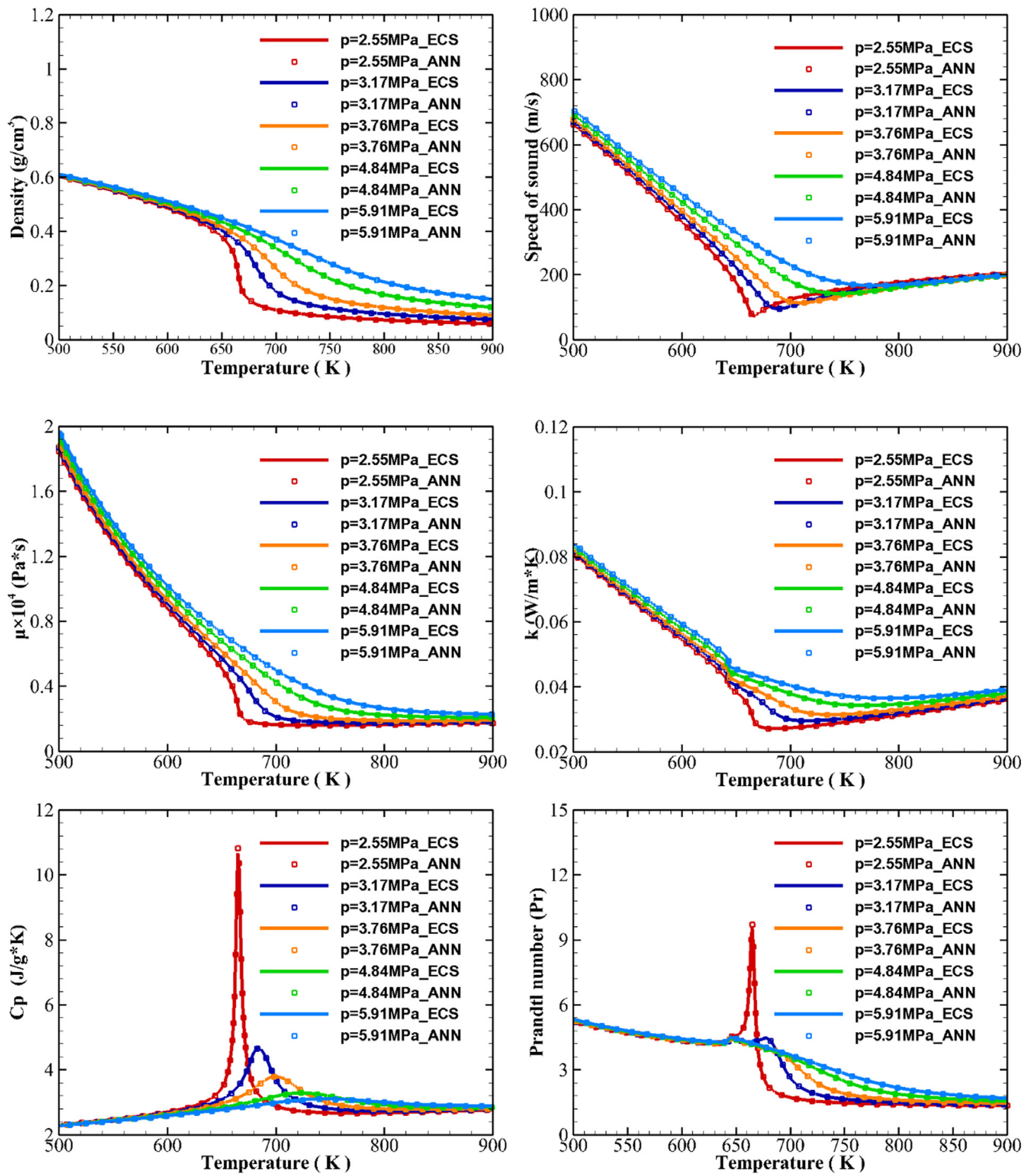

Fig. 4. The comparison between ANN prediction and ECS calculation for the three-component surrogate model of China RP-3 kerosene.

\section{Results and discussion of the ANN model to predict kerosene' properties}

In the above sections, three well trained ANN models have been successfully trained for the prediction of the properties of China RP-3 kerosene under supercritical pressures. The accuracy and efficiency of the models will be evaluated by comparing the ANN predictions with the direct ECS calculations.

\subsection{Accuracy validation}

To evaluate the errors between the predicted results by the trained ANN models and the directly calculated results by the ECS method, five test subsets for each surrogate model of China RP3 kerosene were randomly selected. The to be compared properties are calculated by ANN and ECS respectively for density, sound speed, viscosity, thermal conductivity, specific heat at constant pressure, and Prandtl number. As mentioned before, the five randomly chosen samples have not been used in the previous training. Table 4 summarizes the five test subsets for each surrogate model of kerosene.

The ANN predictions for all the properties were compared with ECS calculations in Figs. 4-6. It can be seen that the ANN predictions match well with the ECS calculations for the three surrogate models of China RP-3 kerosene, even near the critical point at 2.4 MPa. Under supercritical pressures ( $\geq 2.4 \mathrm{MPa}$ ), when the fuel 

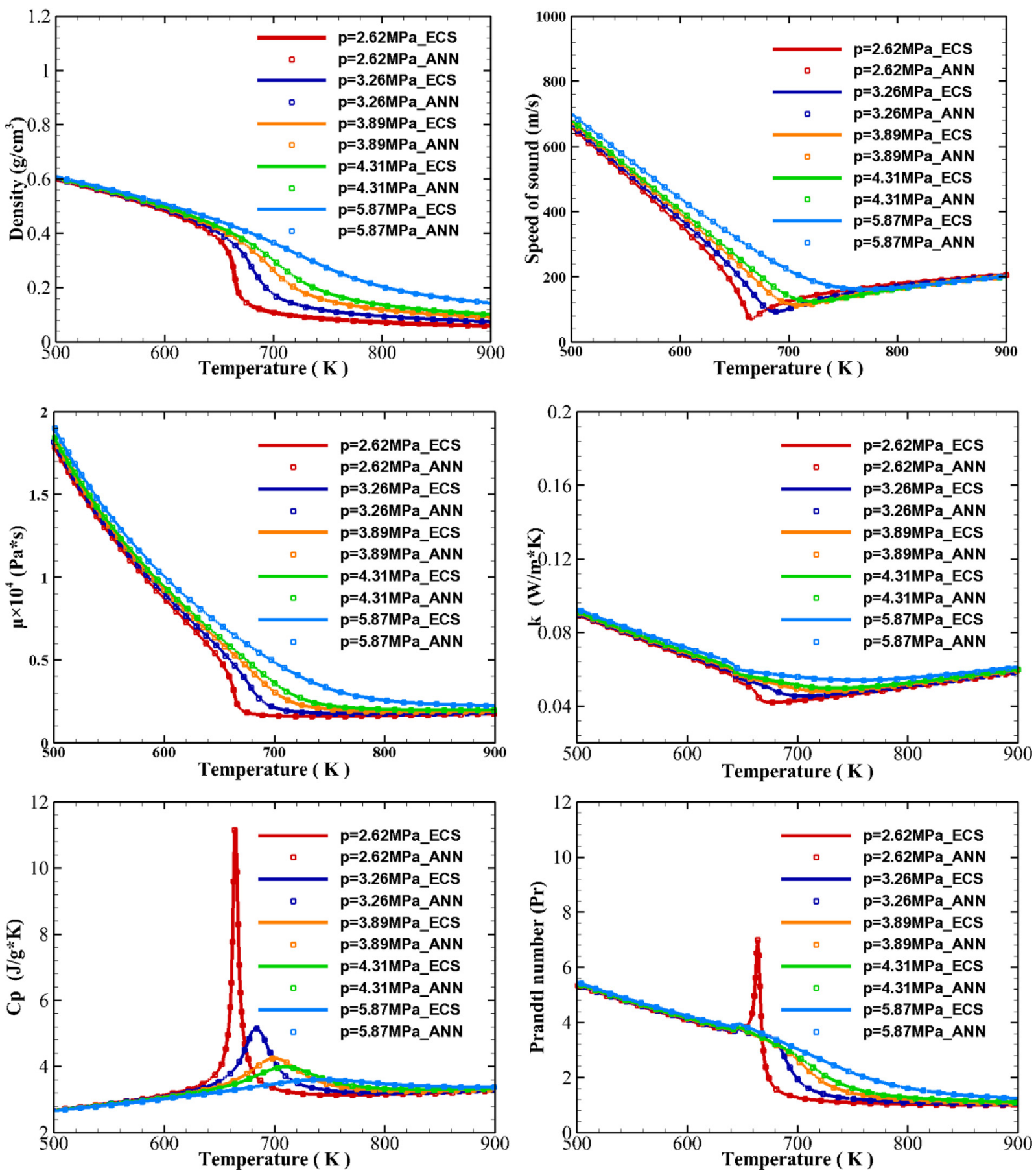

Fig. 5. The comparison between ANN prediction and ECS calculation for the five-component surrogate model of China RP-3 kerosene.

temperature exceeds the pseudo-critical temperature, which varies slightly for different surrogate models, the kerosene in the liquid state will directly transform into the supercritical status without boiling. Near the pseudo-critical temperature region (approximately $660-680 \mathrm{~K}$ ), the properties change dramatically with temperature, especially for $\mathrm{Cp}$ and $\mathrm{Pr}$. The ANN models well reproduce the drastic property changes and peak values.

Fig. 7 shows the correlation coefficient (R) between the ANN predictions and the ECS calculations near the critical region, where larger errors usually occur due to the drastic property changes. The ECS calculations and the ANN predictions match well with each other and are almost identical. It can be seen that all the values of $\mathrm{R}$ are larger than 0.99 and approach unity for each surrogate model. The current accuracy of the trained ANN models is acceptable for further implementation into CFD modelings.

\subsection{Global error analysis}

As shown in Table 5, in addition to the training and validation databases that have been used to develop the ANN models, a new test subset with the size of 497,071 for each surrogate model is constructed to conduct a comprehensive error analysis in the full pressure range of 2.5-6.0 MPa and the temperature range of 300$1000 \mathrm{~K}$, in order to validate further the reliability and accuracy of the trained BP-ANN models. 

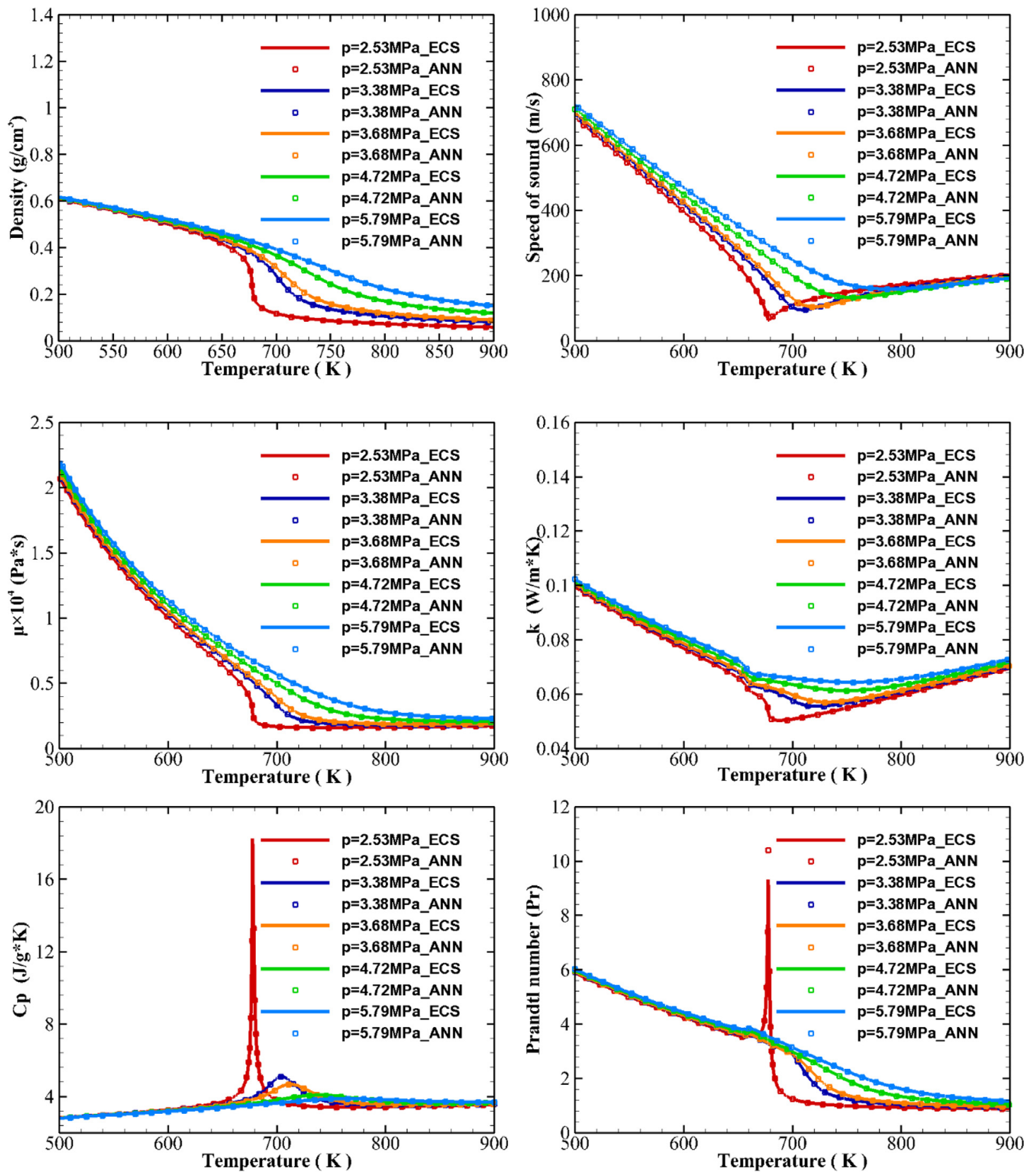

Fig. 6. The comparison between ANN prediction and ECS calculation for the ten-component surrogate model of China RP-3 kerosene.

Table 5

The data used to conduct error analysis.

\begin{tabular}{llll}
\hline Surrogate models & $\mathrm{P}(\mathrm{MPa})$ & $\mathrm{T}(\mathrm{K})$ & The volume of each surrogate mode \\
\hline 3-, 5-, 10-component & $2.5-6.0, \Delta \mathrm{P}=0.05$ & $300-1000, \Delta \mathrm{T}=0.01$ & 497,071 \\
\hline
\end{tabular}

The relative error of each property between the ANN prediction and the ECS calculation is defined as,

Error $=\left|\frac{\text { ANN - SuperTrapp }}{\text { SuperTrapp }}\right| * 100 \%$
The relative error of each property and its probability distribution in the ranges of $0<$ error $\leq 0.1 \%, 0.1 \%<$ error $\leq 0.2 \%, 0.2 \%<$ error $\leq 0.5 \%$, and error $>0.5 \%$ are shown in Figs. $6-8$. Moreover, it can be seen that among the three surrogate models of kerosene, the maximum relative error for all the properties is smaller than 

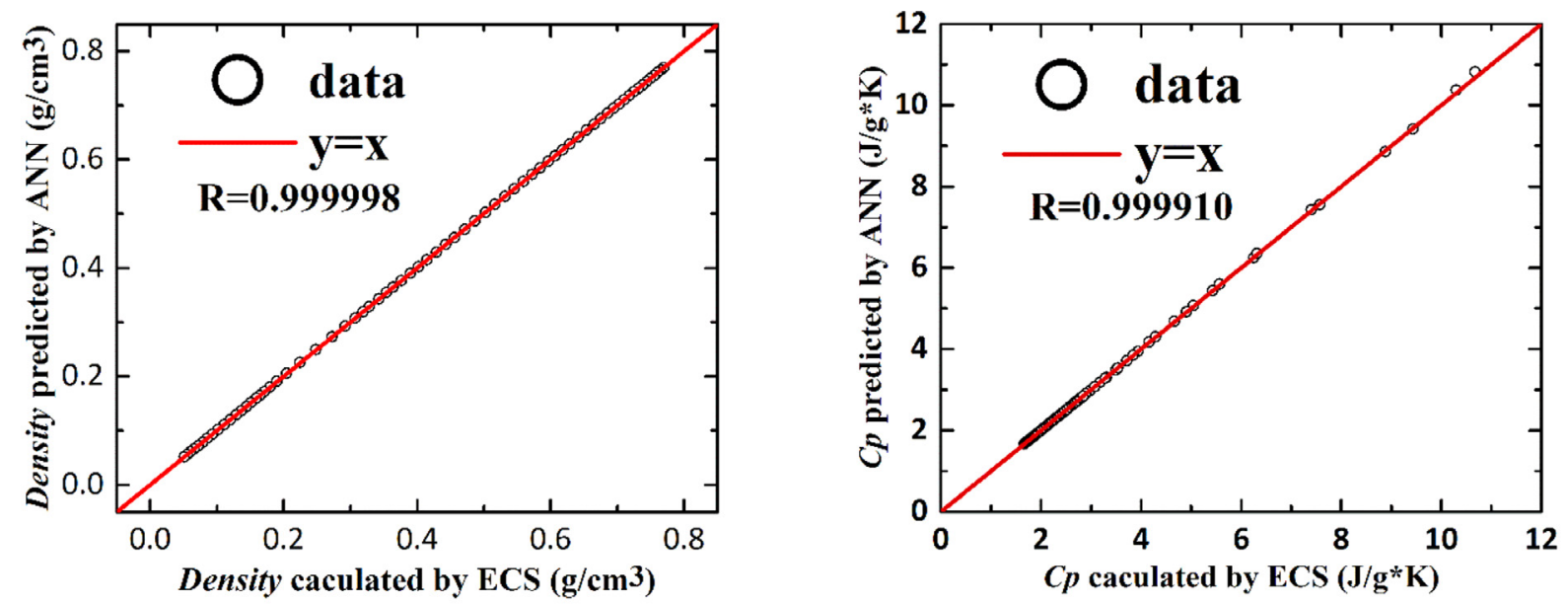

a) 3-component, $\mathrm{P}=2.55 \mathrm{MPa}$
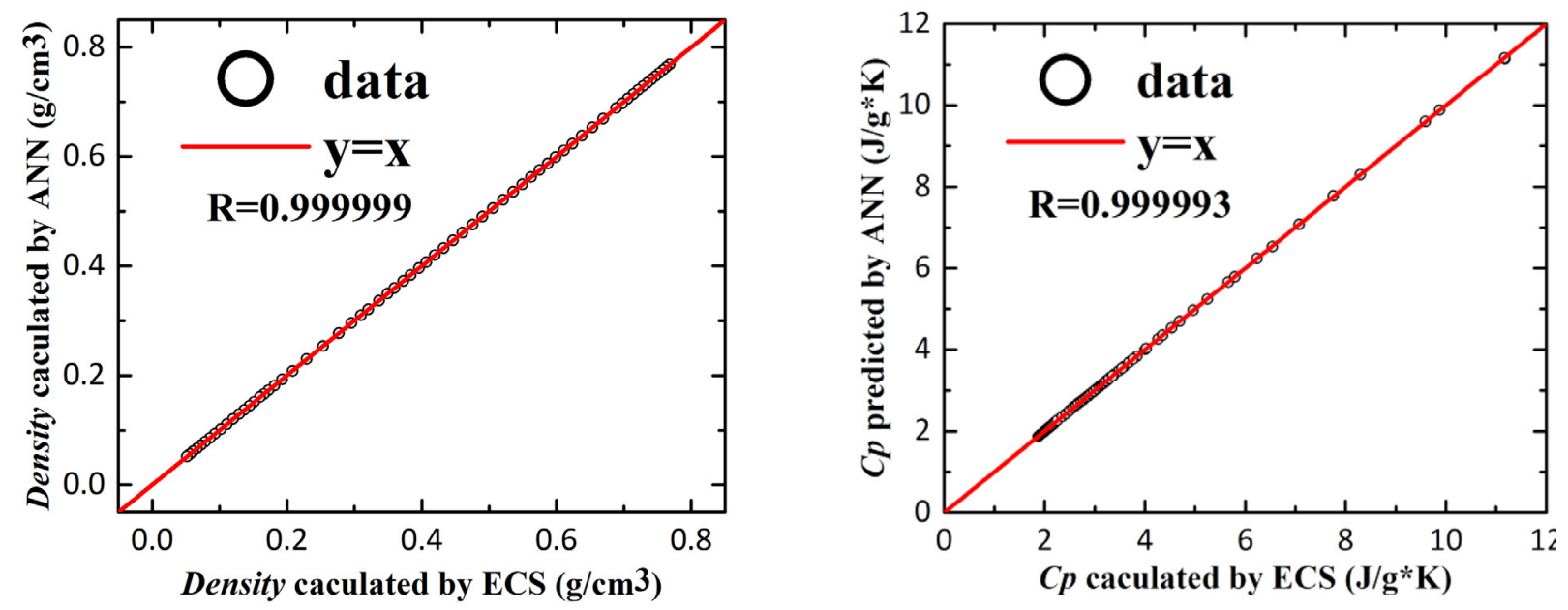

b) 5-component, $\mathrm{P}=2.62 \mathrm{MPa}$
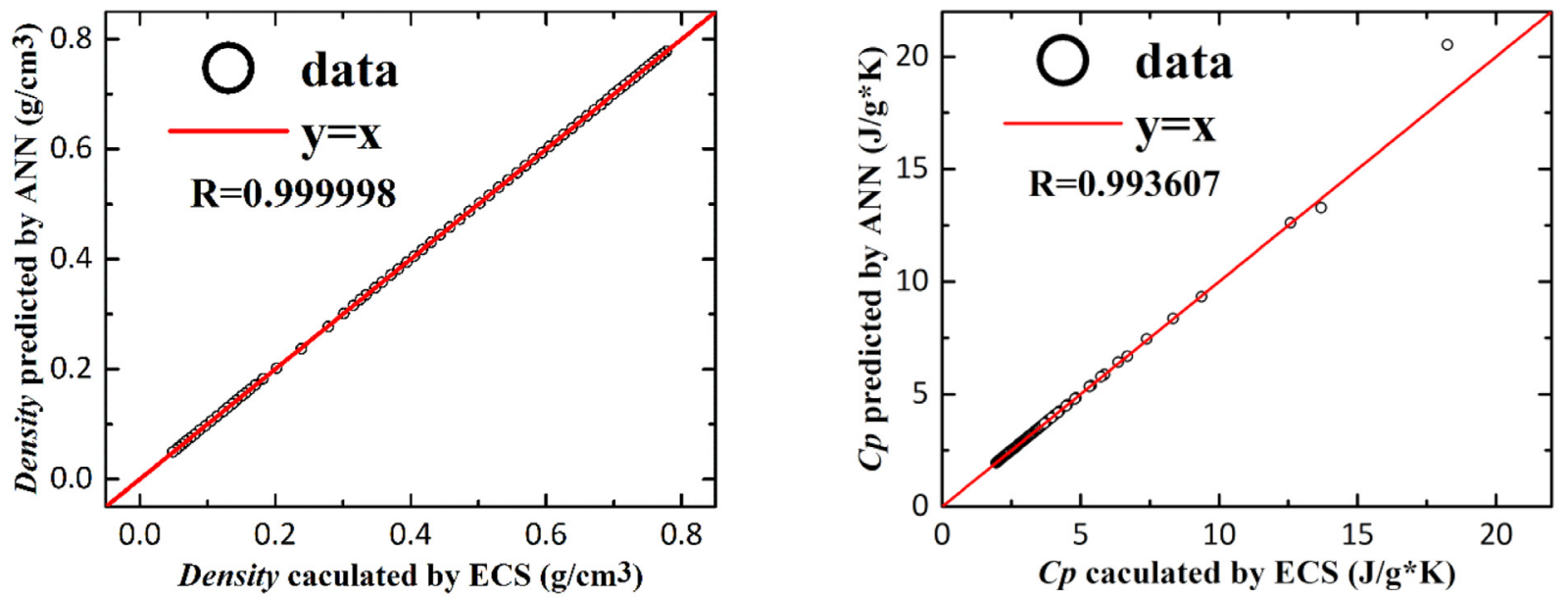

c) 10-component, $\mathrm{P}=2.53 \mathrm{MPa}$

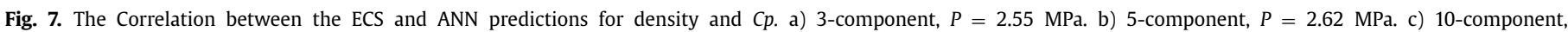
$P=2.53 \mathrm{MPa}$.

$3 \%$ within the targeted pressure range of 2.5-6.0 MPa and temperature range of 300-1000 K. Especially within the pressure range of 2.7-6.0 MPa, the maximum relative error is even lower than $1 \%$ for most of the properties. The large errors mainly exist in a narrow pressure range of $2.5-2.65 \mathrm{MPa}$, probably due to that the properties dramatically change when approaching the critical point (2.4 MPa). Most of the relative errors are smaller than $0.1 \%$, and the amount of relative errors exceeding $0.5 \%$ is far less than $1 \%$.

Comparing the results of the three trained ANN models from Figs. 7 to 9 , it can be found that the proportion with relative er- 


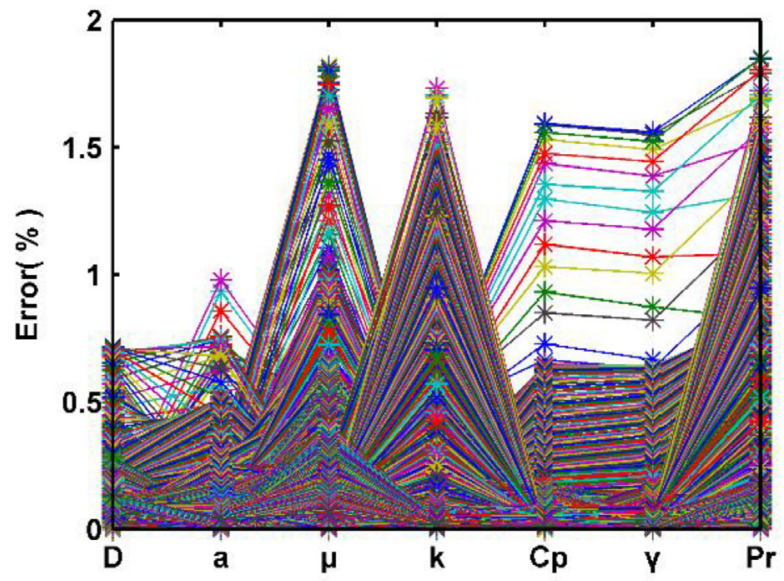

(a)

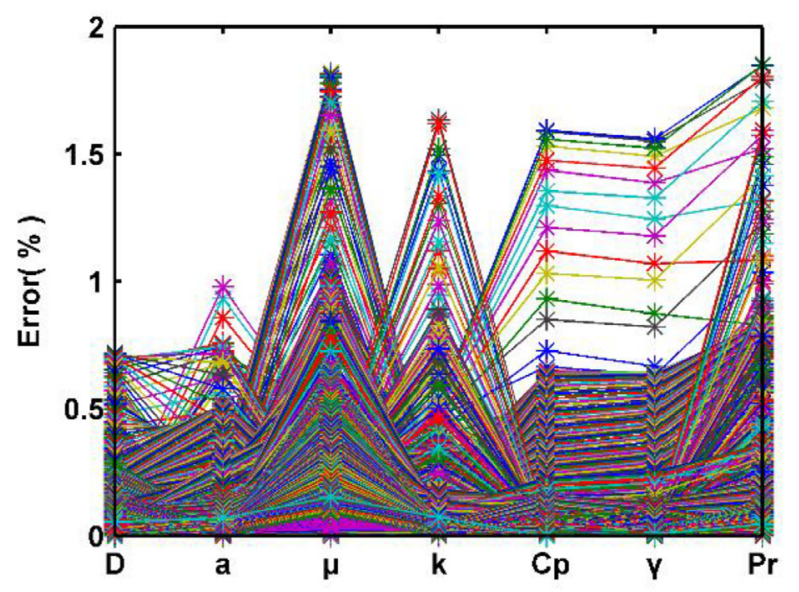

(c)

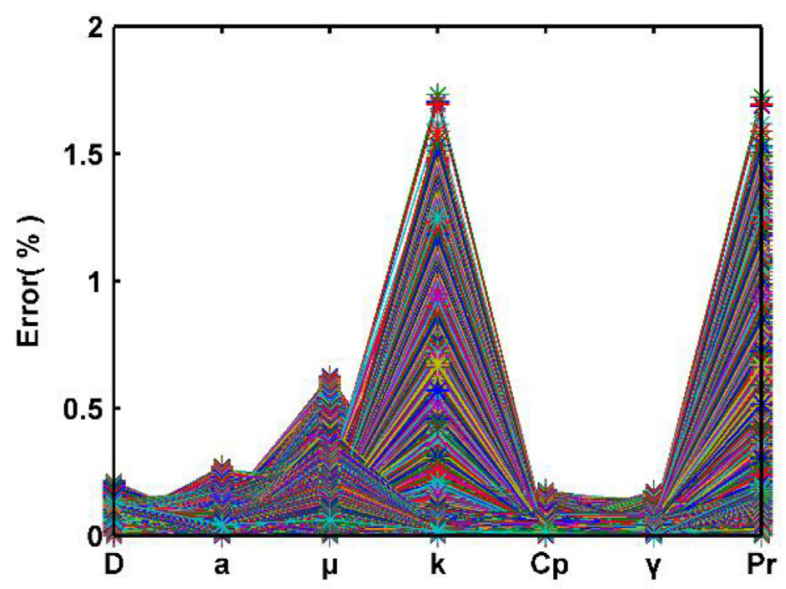

(e)

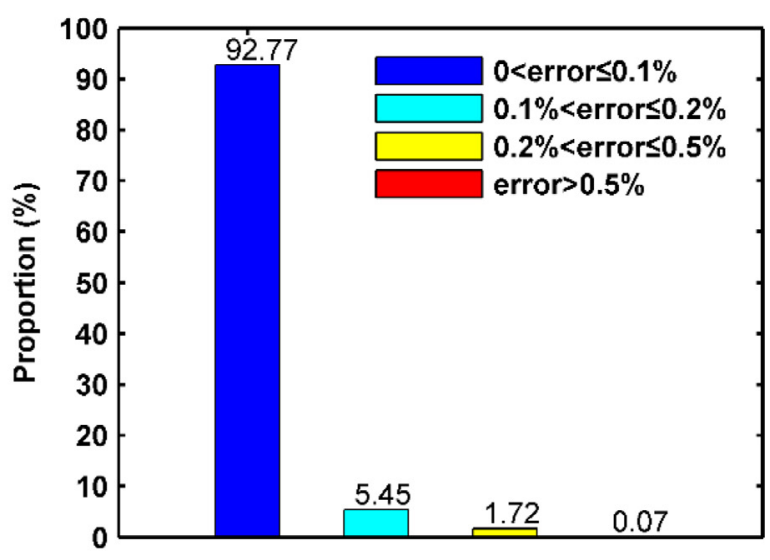

(b)

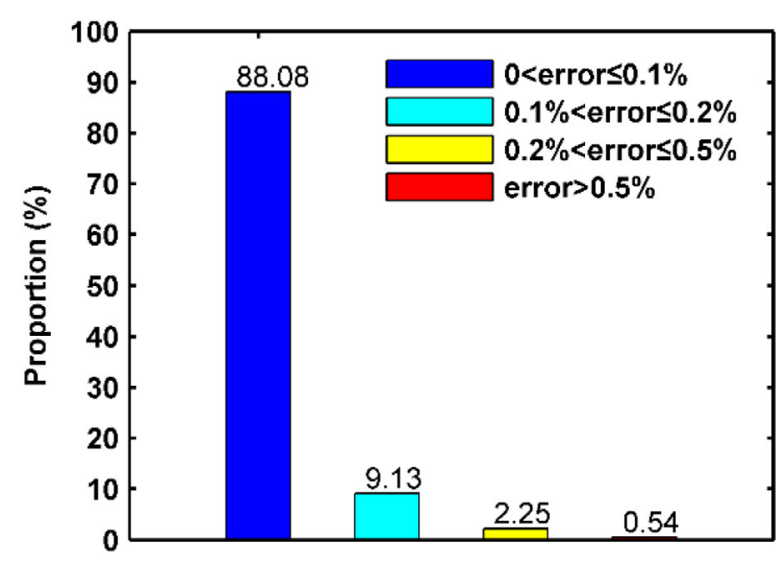

(d)

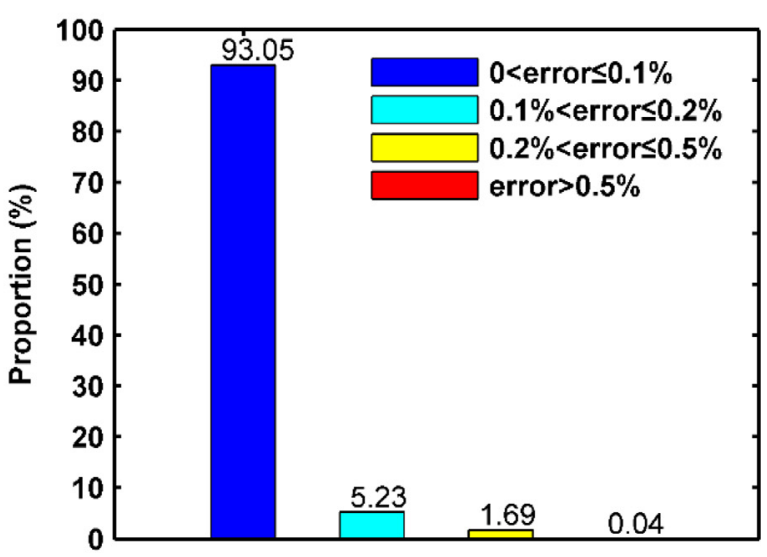

(f)

Fig. 8. The relative error and its distribution for the three-component surrogate model of kerosene, (a) -(b) in 2.5-6.0 MPa, (c)-(d) in 2.5-2.6 MPa, (e)-(f) in 2.7-6.0 MPa.

rors larger than $0.5 \%$ increases with the complexity of the surrogate model. The same observation can be found from the MSE for each surrogate model with the error analysis database. As shown in Table 6 and Fig. 10, the MSE increases with the component number in the surrogate model.

\subsection{Efficiency analysis}

The computational efficiency of the ANN predictions and the ECS calculations are analyzed in this section. As shown in Table 7, three subsets were selected for each surrogate model, including 


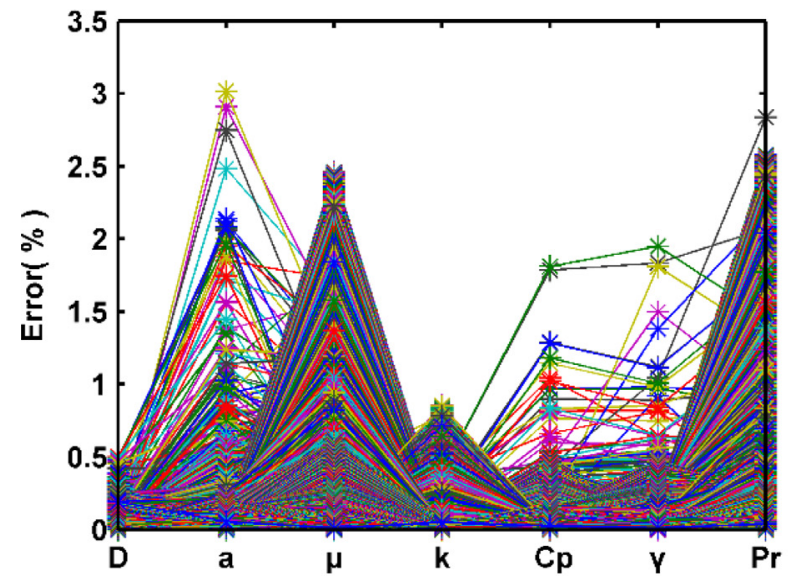

(a)

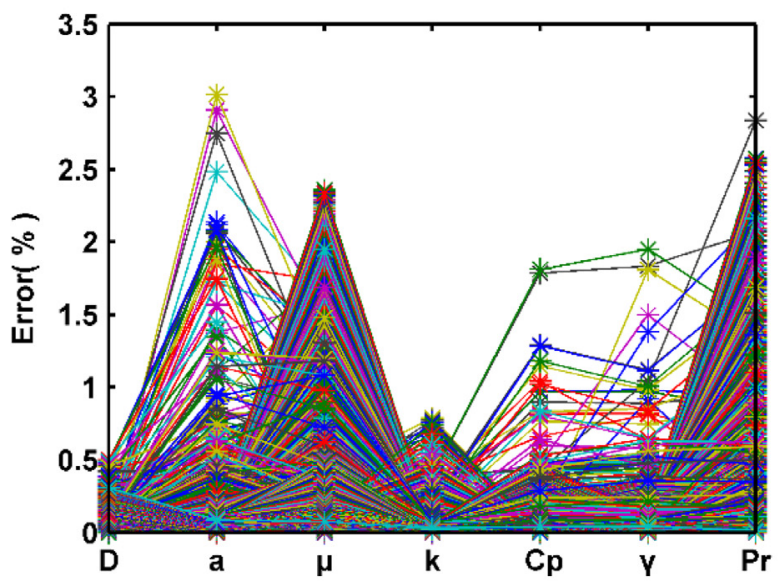

(c)

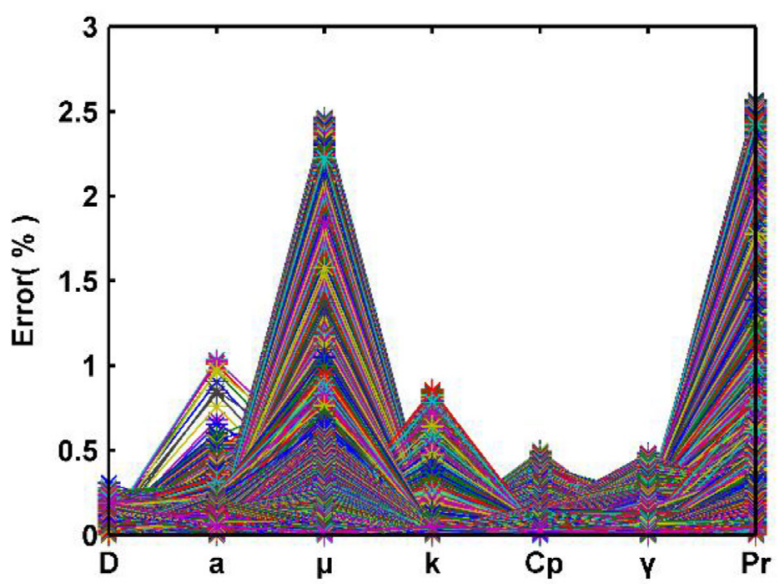

(e)

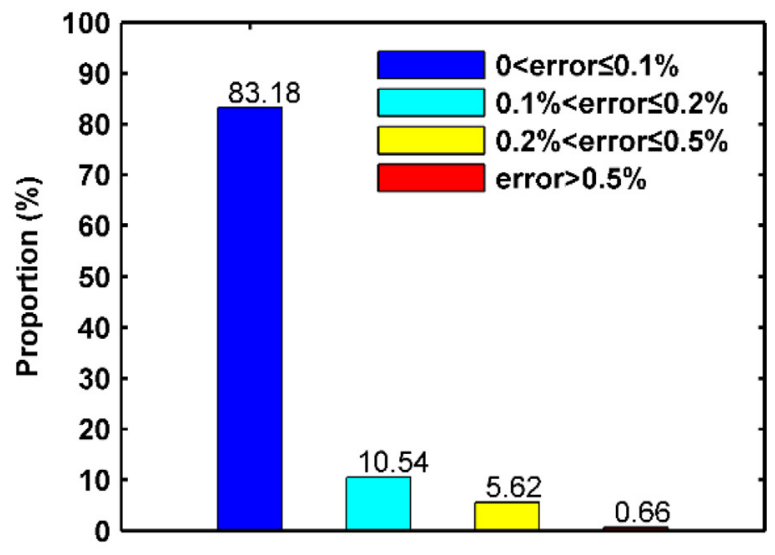

(b)

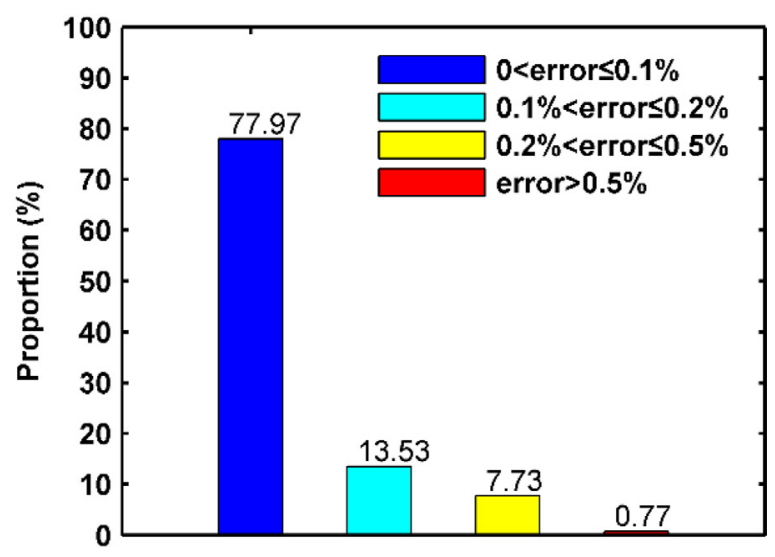

(d)

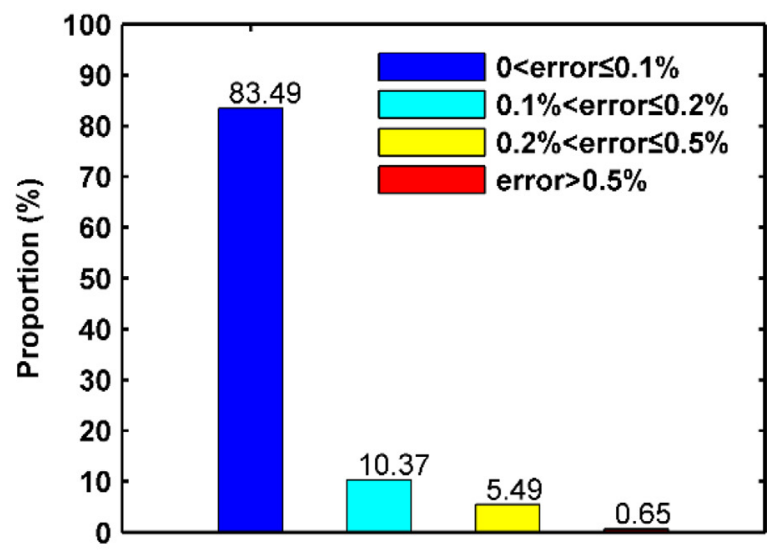

(f)

Fig. 9. The relative error and its distribution for the five-component surrogate model of kerosene, (a) -(b) in 2.5-6.0 MPa, (c)-(d) in 2.5-2.6 MPa, (e)-(f) in 2.7-6.0 MPa.

Table 6

The testing MSE for each surrogate model of China RP-3 kerosene.

\begin{tabular}{ll}
\hline Kerosene & MSE \\
\hline 3-component & $1.98197 \mathrm{e}-006$ \\
5-component & $8.97799 \mathrm{e}-006$ \\
10-component & $1.57077 \mathrm{e}-005$ \\
\hline
\end{tabular}

1) the points in the full range of $2.5-6.0 \mathrm{MPa}$ and $300-1000 \mathrm{~K}$, 2 ) the points away from the pseudo-critical pressure but near the pseudo-critical temperature, i.e., 3-4 MPa and 640-680 K, and 3) the points near the pseudo-critical pressure and the pseudo-critical temperature, i.e., 2.5-2.6 MPa and 640-680 K. For each surrogate model of China RP-3 kerosene, the computational times for the ANN predictions and the ECS calculations are compared in Fig. 11. From the comparisons in Fig. 11, it can be seen that a trained ANN 


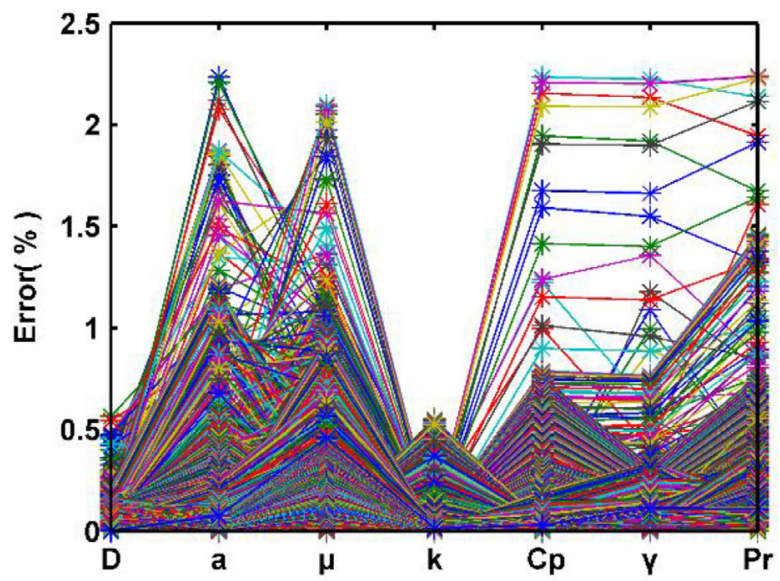

(a)

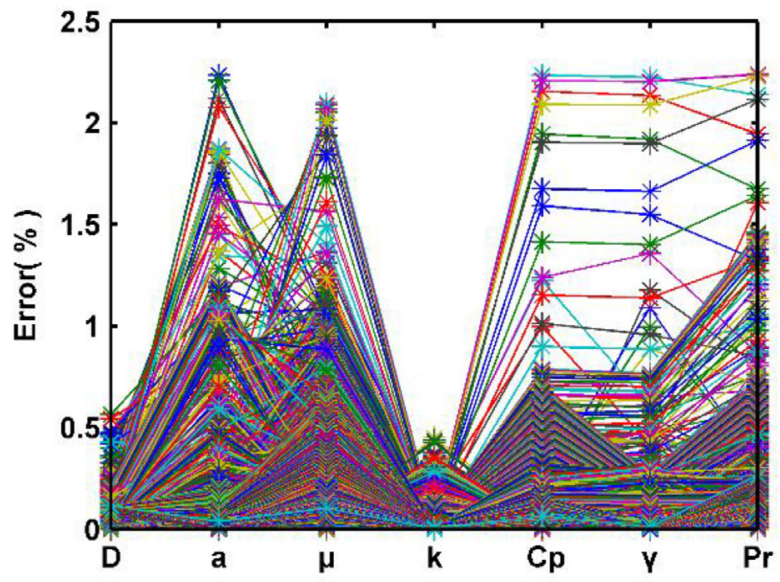

(c)

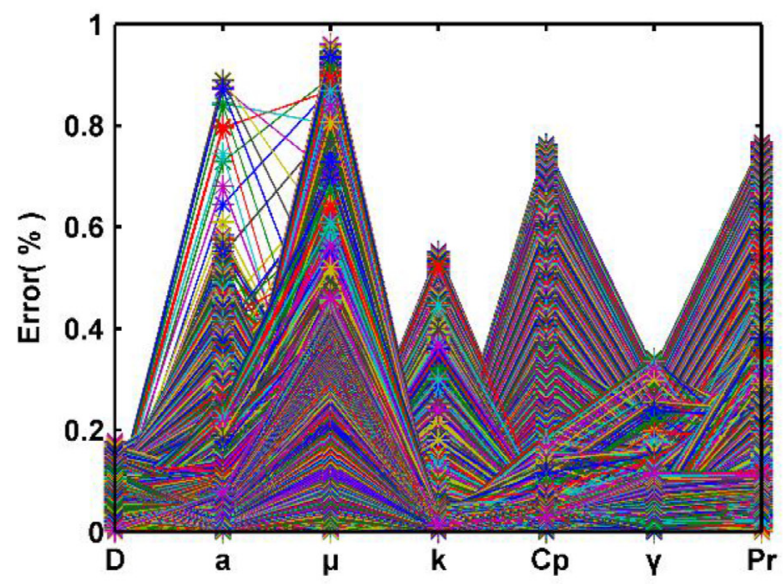

(e)

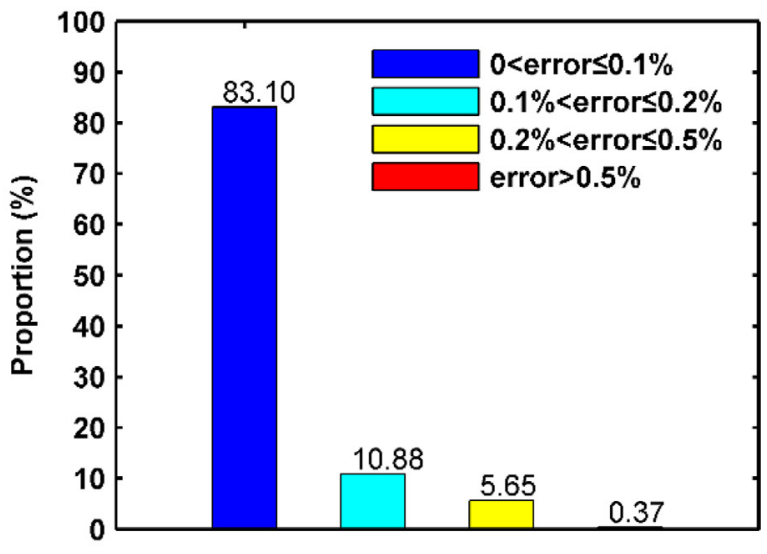

(b)

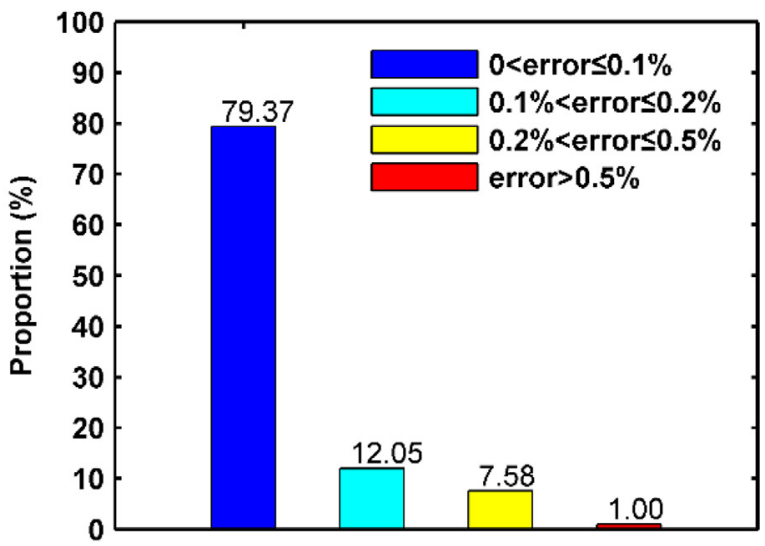

(d)

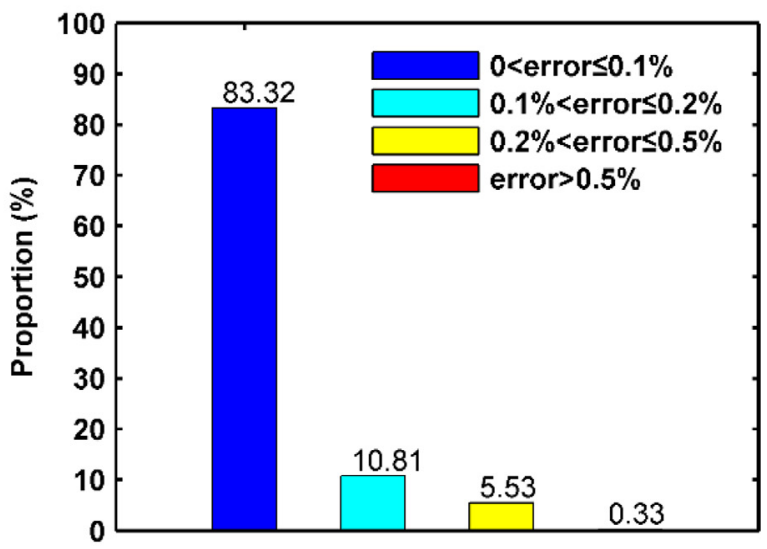

(f)

Fig. 10. The relative error and its distribution for the ten-component surrogate model of kerosene, (a) -(b) in 2.5-6.0 MPa, (c)-(d) in 2.5-2.6 MPa, (e)-(f) in 2.7-6.0 MPa.

model can predict the fluid properties of China RP-3 kerosene at a speed of several orders of magnitude higher than the ECS calculation, especially near the critical point. As the surrogate model becomes more complicated, the ECS calculation usually takes more time; thus, a higher speedup ratio can be achieved by the ANN model.

\section{Performance of the developed ANN model coupled with CFD}

\subsection{Case setup}

To verify the performance of the trained ANN models coupled with the CFD modeling, a jet flow with supercritical China RP-3 


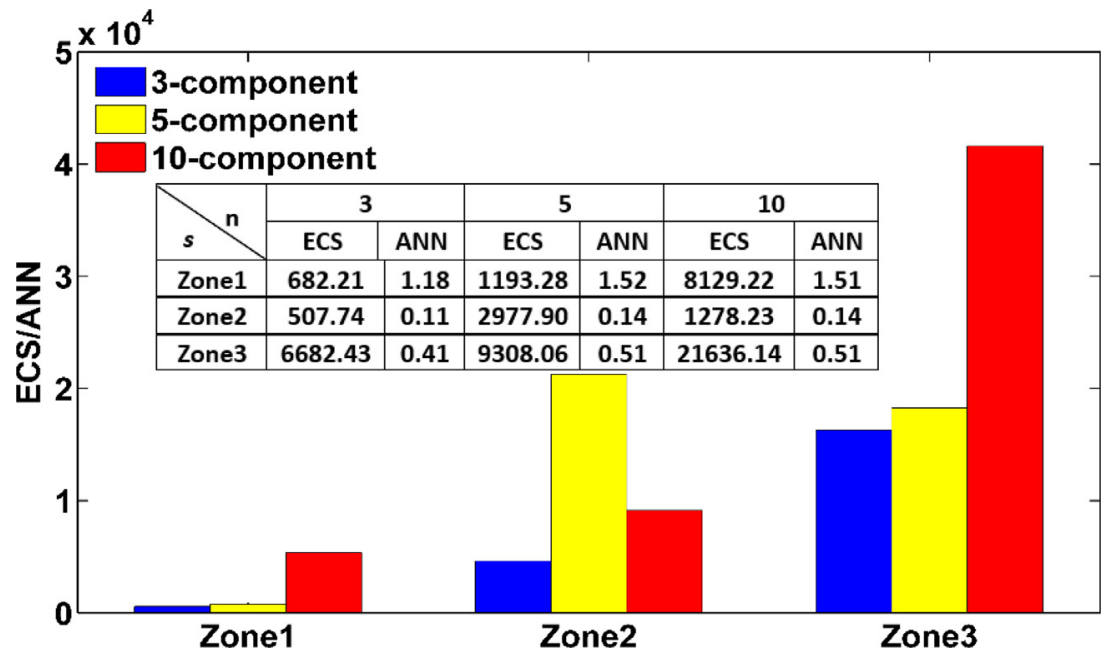

Fig. 11. Efficiency comparison between ANN prediction and ECS calculation.

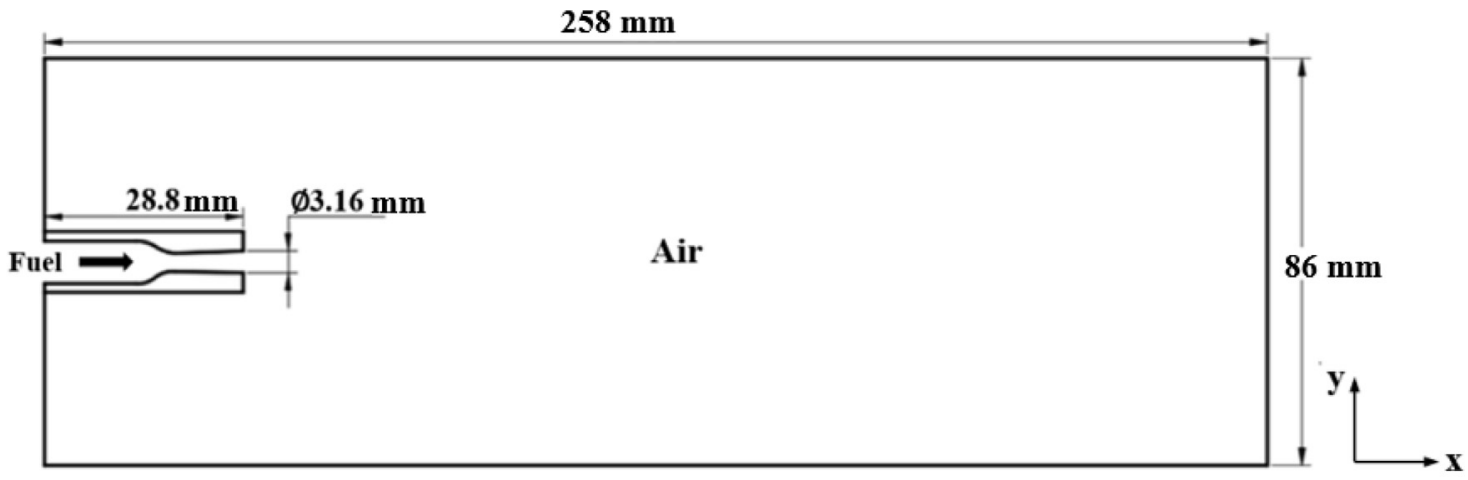

(a)
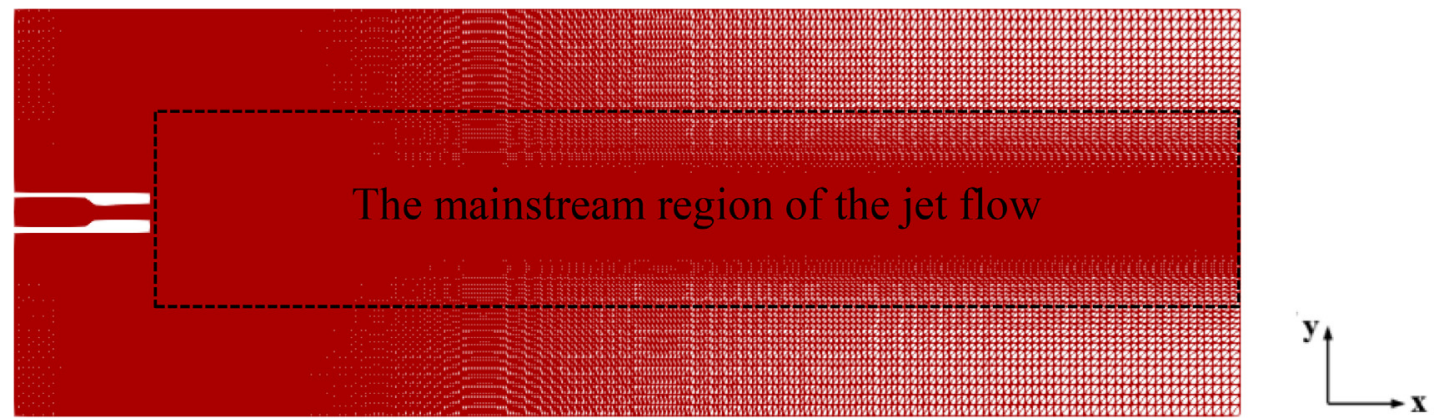

(b)

Fig. 12. (a) schematic of the computational domain including the nozzle, (b) the computational grids.

Table 7

The data used to error analysis.

\begin{tabular}{llll}
\hline Variable & Zone1 & Zone2 & Zone3 \\
\hline $\mathrm{P}(\mathrm{MPa})$ & $2.5 \leq P \leq 6.0$ & $3.0 \leq P \leq 4.0$ & $2.5 \leq P \leq 2.6$ \\
$\mathrm{~T}(\mathrm{~K})$ & $300 \leq T \leq 1000$ & $640 \leq T \leq 680$ & $640 \leq T \leq 680$ \\
\hline
\end{tabular}

kerosene injected into quiescent air is numerically studied. The case has been experimentally studied by Yang et al. [54] by investigating the influence of injection parameters on the structure of the Mach disk. In the CFD modeling, the thermal and transport properties of kerosene are estimated by the ECS method and the developed ANN model is based on the 3-component surrogate [17].

As experimentally studied by Yang et al. [54], the supersonic jet is issued from a convergent-divergent nozzle with $28.8 \mathrm{~mm}$ in height and $3.16 \mathrm{~mm}$ in the exit diameter, as schematically shown in Fig. 12(a). The hexahedral computational domain has dimensions of $258 \times 86 \times 86 \mathrm{~mm}$ in the $\mathrm{x}, \mathrm{y}$, and $\mathrm{z}$ directions, respectively. To evaluate the grid sensitivity, four sets of unstructured mesh are tested respectively with $6.19,14.24,21.42$, and 35.17 million cells. In all the mesh sets, cells along the main jet shear layer are refined as shown in Fig. 12(b).

The kerosene is initially injected at supercritical status with a temperature of $750 \mathrm{~K}$ and a pressure of $2.9 \mathrm{Mpa}$, which are larger than the critical values. The quiescent air is the mixture of nitrogen in 76.699 mass\% and oxygen in 23.301 mass\%, whose initial temperature, pressure, density, and velocity are all uniform, i.e., $\mathrm{T}_{\infty}=298 \mathrm{~K}, \mathrm{P}_{\infty}=101,325 \mathrm{~Pa}, \rho_{\infty}=1.17 \mathrm{~kg} / \mathrm{m}^{3}, \mathrm{U}_{\infty}=0$. The inflow at the nozzle inlet is subsonic; accordingly, the stagnation condition is employed for the temperature and the pressure, while 

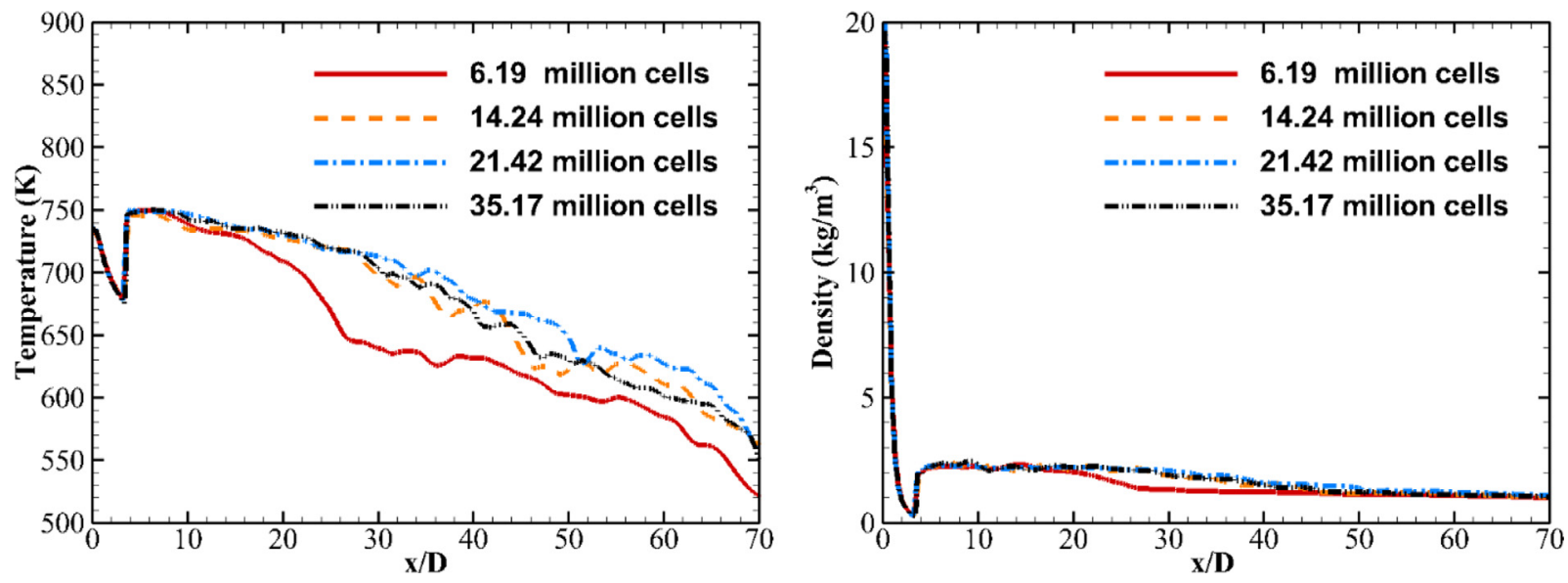

Fig. 13. Grid sensitivity of the time-averaged temperature and density for the jet flow, data obtained from the central axis for different mesh resolutions.

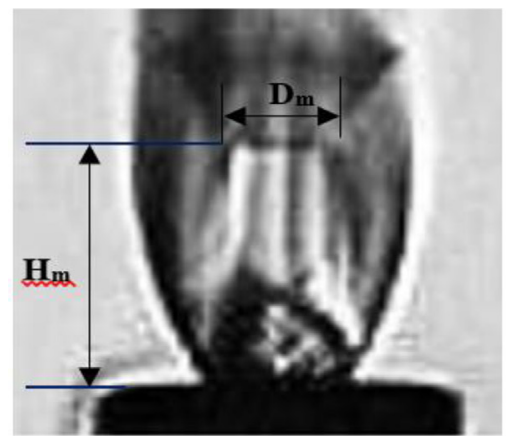

Experiment

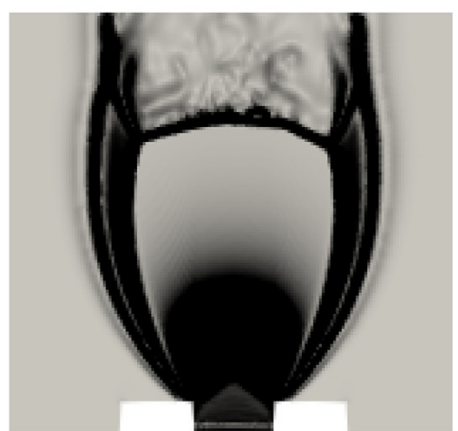

ECS

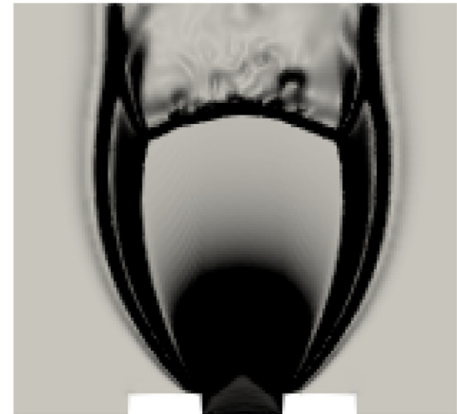

ANN

Fig. 14. Comparison of the Mach disk structure between experiment and current LES's simulations nearing the nozzle exit by ECS and ANN respectively.

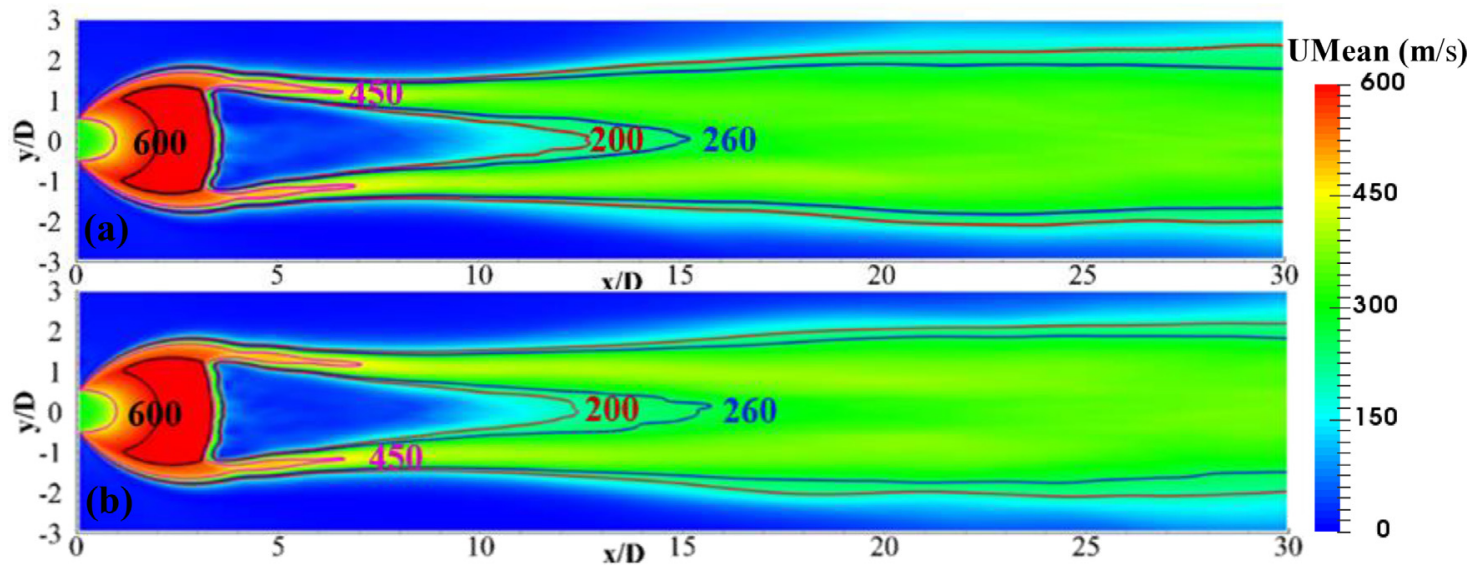

Fig. 15. The time-averaged contours of velocity and, which were simulated by ANN and ECS respectively: (a) ECS, (b) ANN.

a zero-gradient condition is used for velocity. For the outlet and the lateral boundaries, the zero-gradient condition is applied for the outflow flux, while the ambient conditions are specified should backflow occur. No-slip and adiabatic conditions are used for all the walls.

Large Eddy Simulation (LES) in this study is conducted by an in-house developed Finite Volume Method (FVM) based compressible flow solver AstroFoam [55], which is density-based and extended from the rhoCentralFOAM solver distributed with the opensource CFD package OpenFOAM [56]. AstroFoam has been successfully applied in many previous studies [55,57-59]. The subgridscale (SGS) turbulence effect is closed by the dynamic subgrid ki- netic energy model (DKEM) [60]. The inviscid convection is solved by the semidiscrete K-T scheme [61]. The scale-selective discretization (SSD) scheme [62] is used to reconstruct the primitive values at faces with a third-order spatial accuracy. Under the temperature close to the room temperature $(298 \mathrm{~K})$ and standard atmospheric pressure, the real-gas behavior of nitrogen and oxygen is close to the ideal gas. Therefore, the property of nitrogen and oxygen is predicted by the ideal gas law to reduce the computational cost. Notably, the real-gas effect of kerosene is accounted for by the ECS principle and the ANN method. The flow-through time (FTT) for the jet washing out the computational domain is about $1 \mathrm{~ms}$, and the data-sampling duration is $10 \mathrm{FTT}=0.01 \mathrm{~s}$. 
(a) ECS, (b) ANN.

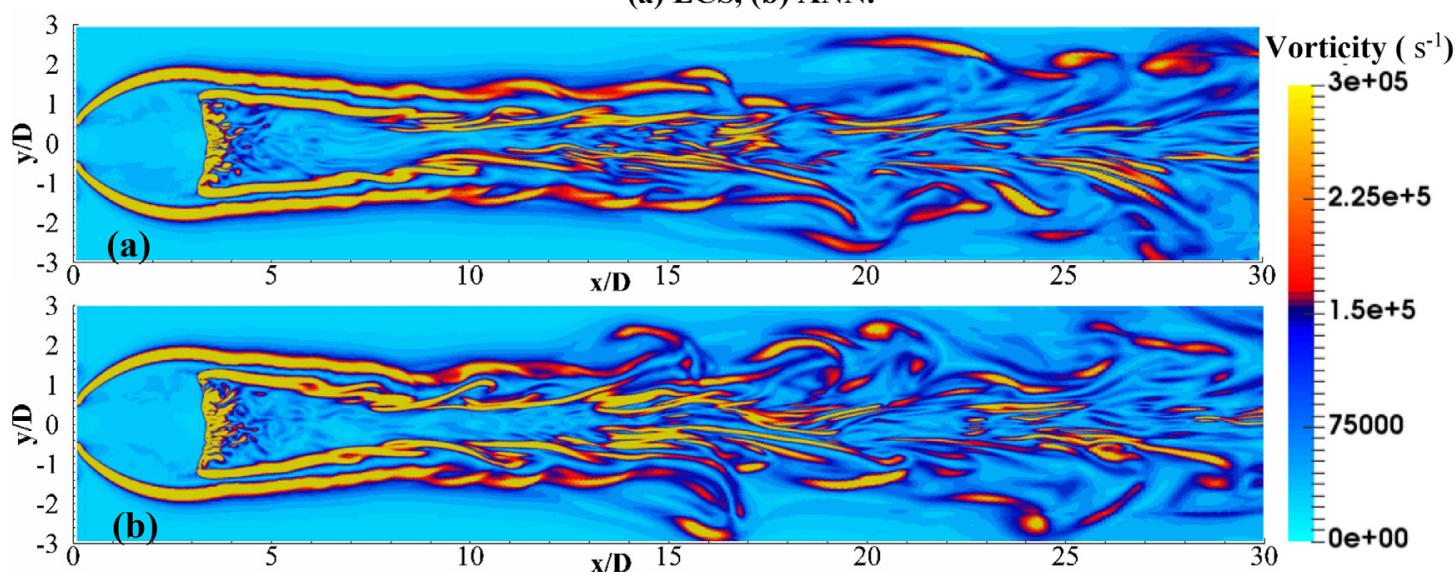

Fig. 16. The magnitude of instantaneous vorticity field $|\boldsymbol{\Omega}|=|\nabla \times \overrightarrow{\boldsymbol{V}}|$ at the plane of $z=0$, which were simulated by ANN and ECS respectively: (a) ECS, (b) ANN.

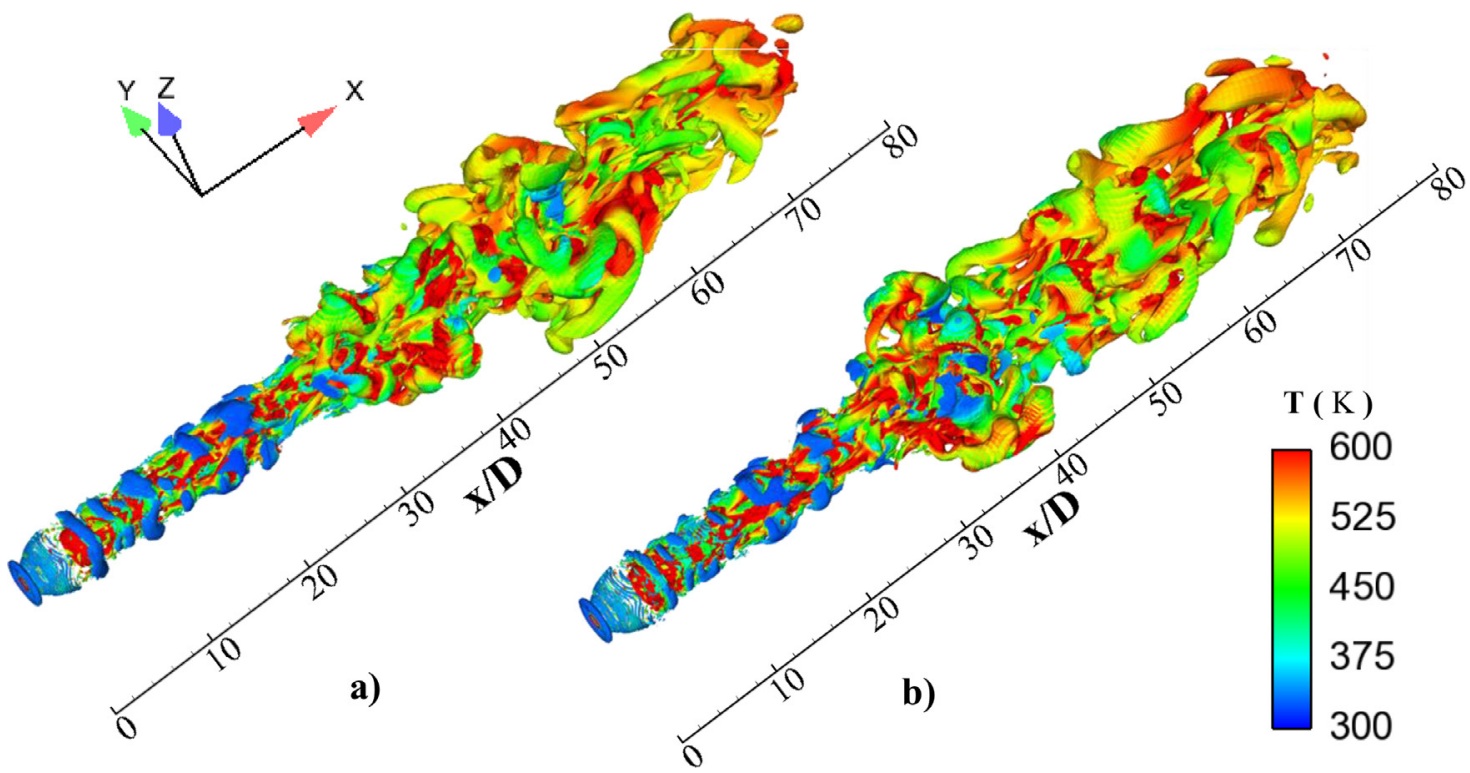

Fig. 17. The three-dimensional instantaneous isosurfaces of the Q-criterion ( $\mathrm{Qc}=1.6 \times 10^{6} s^{-2}$ ) colored by time-averaged temperature, a) ECS, b) ANN.

\subsection{Accuracy analysis of ANN coupled with CFD}

A grid convergence study based on $6.19,14.24,21.42$, and 35.17 million cells is conducted to establish the fidelity of the LES results. The mean axial temperature and density profiles in Fig. 13(a) and (b) are visually similar for the mesh sets with cells more than 14.24 million. Especially for the density profiles, the results with 21.42 and more million cells have indistinguishable discrepancies. For the temperature profiles, the discrepancies between the results based on the mesh with 14.24 million cells and the finer meshes fall within $5.1 \%$, while the maximum error between the results based on the 35.17 million cells and the 6.19 million cells is $10.01 \%$. In the following analysis, the results based on the 35.17 million cells are used.

Fig. 14 compares the structure of the Mach disk between the schlieren image and the LES results near the nozzle exit. As summarized in Table 8 , the predicted height $H_{m}$ and width $D_{m}$ of the Mach disk by the ANN model and the ECS principle are almost identical, with the relative errors of $0.61 \%$ and $1.4 \%$ respectively for $H_{m}$ and $D_{m}$. Compared with the experiment, the height of the Mach disk predicted by both the ANN model and the ECS principle is slightly larger with a relative error of $20 \%$, while the predicted diameter of the Mach disk agrees well. (See Fig. 14).
Table 8

The comparison of Mach disk structure ( $\mathrm{mm})$.

\begin{tabular}{llll}
\hline Kerosene & Experiment & ECS & ANN \\
\hline$H_{m}$ & 8.10 & 9.76 & 9.82 \\
$D_{m}$ & 4.59 & 4.67 & 4.74 \\
\hline
\end{tabular}

As compared in Fig. 15, the time-averaged contours of velocity predicted by ANN and ECS are visually similar. Both the maximum penetration depth of the jet is around 2.5D for (a) and (b). The positions of the contour lines at $600(\mathrm{~m} / \mathrm{s}), 450(\mathrm{~m} / \mathrm{s}), 260(\mathrm{~m} / \mathrm{s}), 200$ $(\mathrm{m} / \mathrm{s})$ are similar. Fig. 16 shows two instantaneous images of the vorticity magnitude for the jet flow, which were simulated by ECS and ANN, respectively. As can be seen, the flow remains laminar, and there is little difference in the region of $x<13.5 \mathrm{D}$ predicted by ECS and ANN. Further downstream, these coherent vortex rings structures rollup because of the instability of the jet shear layer. The initial position where instability occurs is 15D and 14D for ECS and ANN. Though the predicted locations of large-scale vortexes are slightly different, the development process and the degree of instability of vortexes are very similar for ECS and ANN.

Fig. 17 shows the three-dimensional instantaneous isosurfaces of the Q-criterion, defined as $\mathrm{Qc}=-\frac{1}{2}\left(\Omega_{i h} \Omega_{i j}-S_{i j} S_{i j}\right)$ with $\mathrm{S}$ and 
(a)

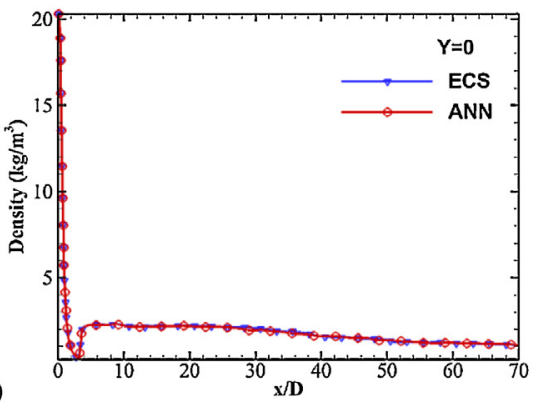

(c)

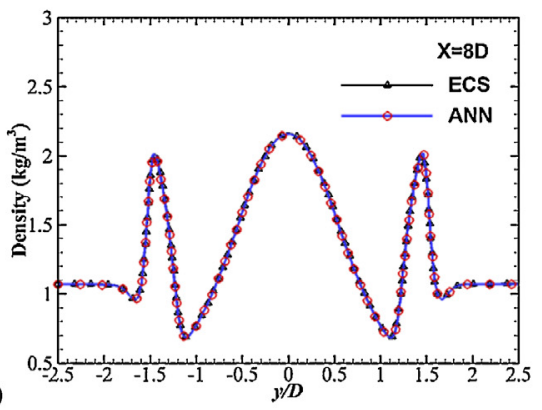

(e)

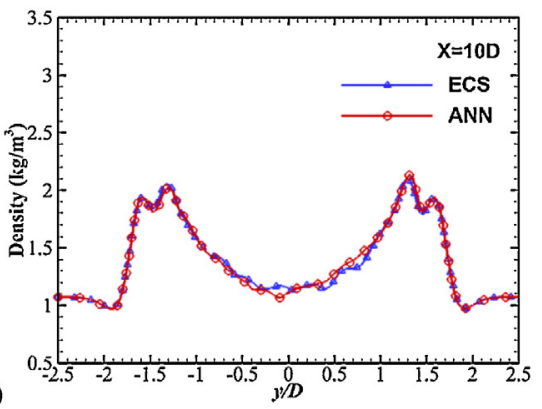

(g)

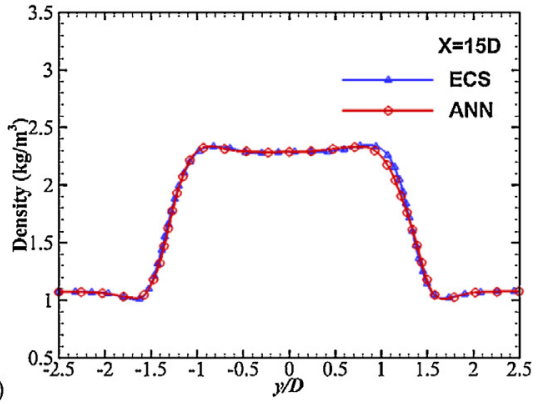

(i)

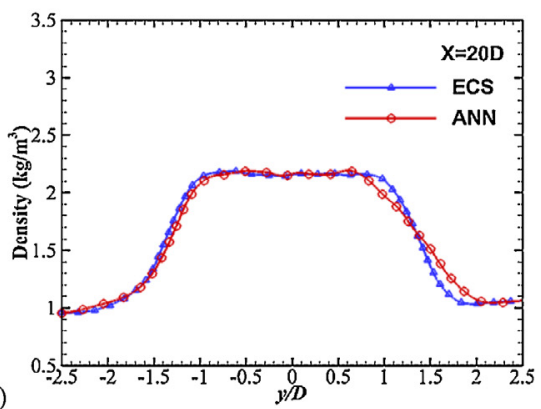

(b)

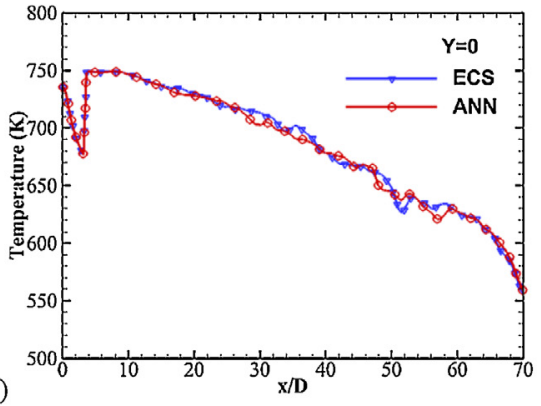

(d)

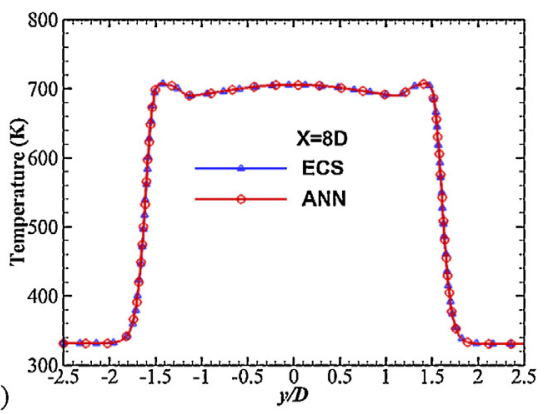

(f)

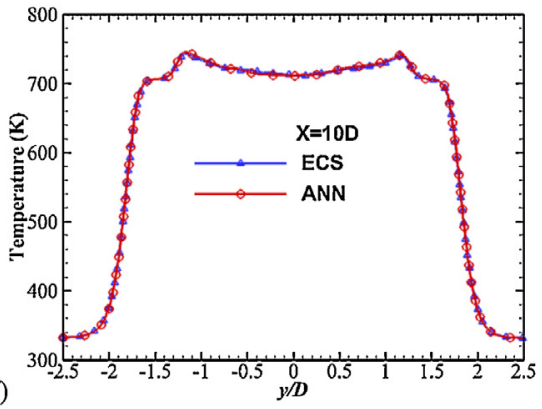

(h)

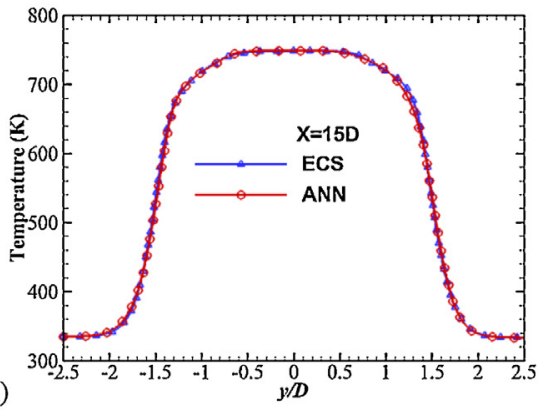

(j)

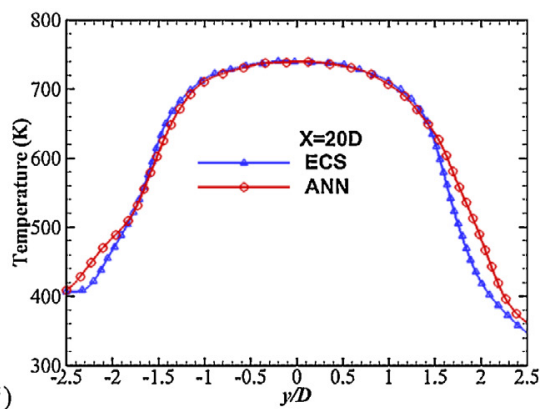

Fig. 18. The comparison between the ANN prediction and the ECS calculation for time-averaged density and temperature on (a)-(b) the axis, and the radial at (c)-(d) $x=8$ D, (e)-(f) $x=10 \mathrm{D}$, (g)-(h) $x=15 \mathrm{D}$, and (i)-(j) $x=20 \mathrm{D}$ Computational efficiency analysis. 


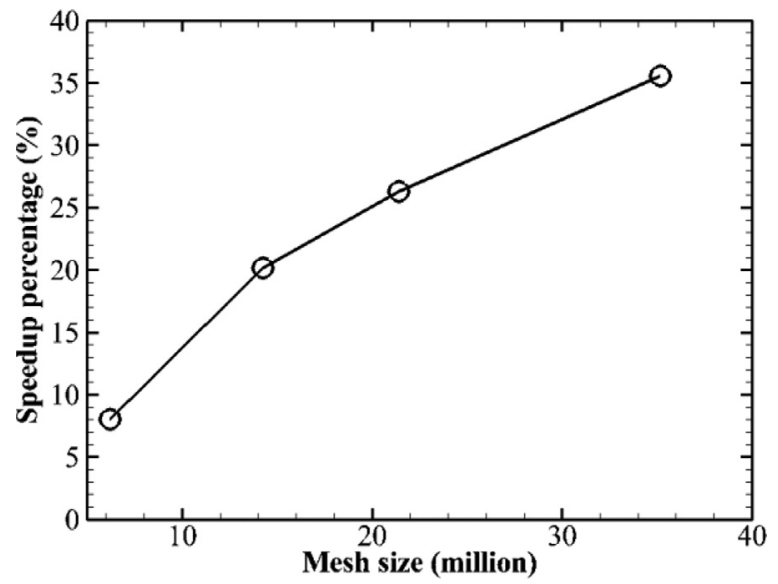

Fig. 19. Speedup percentage of ANN model with the mesh size.

$\Omega$ the strain rate tensor and rotation rate tensor, respectively. Ring vortices are initially formed at the jet root due to the KelvinHelmholtz (K-H) instability and gradually transform into $\Omega$-shape vortices with legs attached to the jet core when moving toward the downstream direction. The vortex stretching term mainly occurs along the outer and inner shear layers, where the sizeable coherent $\Omega$ vortices are finally broken into smaller vortex structures. The heights of the jet potential core indicated by the initial broken of the $\Omega$ vortices are both around $x=32 \mathrm{D}$ in the ANN and ECS predictions.

Fig. 18 compares the time-averaged axial and radial profiles of density and temperature between the ANN predictions and the ECS calculations. As can be seen, the ANN predictions agree well with the ECS calculations. Slightly larger deviations occur for $x=20 \mathrm{D}$, probably because the larger jet instability in the downstream magnifies the difference. The average errors are $1.98 \%$ and $1.79 \%$, while the maximum errors are $8.38 \%$ and $9.27 \%$ respectively for the density and the temperature. The accuracy of the ANN model is overall acceptable for the small average discrepancies.

To accurately compare the efficiency of the ANN model, all the modeling of the supercritical jet flow was conducted on the same parallel scale using 300 processors. The time spent in the parallel communicaiton has been included in the computation time. As shown in Fig. 19, the speedup ratios of the ANN model are $8 \%$, $20.15 \%, 26.31 \%$, and $35.52 \%$ for the mesh sizes of 6.19 million, 14.24 million, 21.42 million, and 35.17 million. Generally, the computation efficiency is improved with the growth of the mesh size. For the ECS case and the ANN case based on 35.17 million cells, the CPU time for a single time step is $45.60 \mathrm{~s}$ and $29.40 \mathrm{~s}$, and a total of 120,000 and 77,400 CPU hours are respectively required to finish the same flow-through times (FTTs). Though the training procedure is time-consuming, the developed ANN model has exhibited great advantage in computational efficiency, especially for largescale modeling.

Comparing Figs. 11 and 19, it can be seen that the speedup ratio of ANN is far smaller when it is coupled with CFD. This is because, 1) the modeling of flow and turbulence takes additional cost; 2) the application of the real-gas calculation is restricted to the fuel stream, while ideal gas law is applied for the rest flow region. Despite that limitation, a considerable improvement in computational efficiency has already been achieved by using the developed ANN model Fig. 10.

\section{Conclusion}

In this study, efficient yet accurate ANN models were developed to predict the fluid properties of China RP-3 kerosene at supercrit- ical pressure, making CFD modeling taken into account of real-gas effects computationally-affordable. Three ANN models for different kerosene surrogate models have been successfully trained by using the backpropagation (BP) algorithm, adaptive learning rate, and adaptive momentum method. The training database for real gas properties is calculated based on the principle of Extended Corresponding State (ECS) for each kerosene surrogate model. For all the surrogate models, the largest Training MSE (lowest root mean square error) and Validation MSE are as small as 6.52347e-08 and 4.9839e-04.

A comprehensive error analysis between ANN prediction and ECS calculation in the full range has been conducted. Comparisons for five randomly-selected sample sets show that the ANN predictions agree well with the ECS calculations even at the points approaching the critical pressure and the critical temperature, where usually drastic property changes occur. The correlation coefficients (R) between the ANN prediction and ECS calculation at lower pressure are all larger than 0.99 and approaching to the perfect fitting status with $R=1$. The largest relative error is lower than $3 \%$, the proportion of the relative errors below $0.1 \%$ is higher than $80 \%$, and the proportion of the relative errors above $0.5 \%$ is no more than $1 \%$. Both the number of hidden layers and the neuron number in each hidden layer increase with the complicity of the surrogate model.

The computational efficiency of the ANN model and the ECS method was compared. The trained ANN model predicts the fluid properties of kerosene at a speed of several orders (arriving at $10^{4}$ ) higher than the ECS calculation, especially near the critical point. The trained ANN model was further coupled with the CFD modeling of a kerosene jet to validate its performance. The ANN model shows high similarity compared with the ECS calculations. The computational efficiency increases with the mesh size, and a high speedup ratio above $35.52 \%$ was achieved at a 35.17 -millioncell mesh.

This study successfully developed and validated the accuracy and computational efficiency of the ANN method, which shows the feasibility of the ANN model in replacing the computationallycostly real-gas property calculation. The training and CFD-coupling framework for kerosene in this study can be further extended for the property evaluations of other materials.

\section{Declaration of Competing Interest}

The authors declare that they have no known competing financial interests or personal relationships that could have appeared to influence the work reported in this paper.

\section{CRediT authorship contribution statement}

Bo Li: Data curation, Formal analysis, Writing - original draft. Yachao Lee: Data curation. Wei Yao: Conceptualization, Formal analysis, Writing - original draft. Yang Lu: Formal analysis. Xuejun Fan: Conceptualization.

\section{Acknowledgments}

The project was supported by Training Program of the Major Research Plan of the National Natural Science Foundation of China (grant no. 91641110), National Key Research and Development Program of China (2019YFB1704200), and the Open founding of National Key Laboratory of Science and Technology on Aero-Engine Aero-Thermodynamics (grant no. 6142702180307). The authors are also grateful to the National Supercomputer Center in Tianjin for providing the computational resource. 


\section{References}

[1] Cui M, Mei J, Zhang B-W, Xu B-B, Zhou L, Zhang Y. Inverse identification of boundary conditions in a scramjet combustor with a regenerative cooling system. Appl Therm Eng 2018;134:555-63.

[2] Tao Z, Cheng Z, Zhu J, Lin D, Wu H. Large eddy simulation of supercritical heat transfer to hydrocarbon fuel. Int J Heat Mass Transf 2018;121:1251-63.

[3] Li X, Huai X, Cai J, Zhong F, Fan X, Guo Z. Convective heat transfer characteristics of China RP-3 aviation kerosene at supercritical pressure. Appl Therm Eng 2011;31(14-15):2360-6.

[4] Zhong F, Fan X, Yu G, Li J, Sung C-J. Heat transfer of aviation kerosene at supercritical conditions. J Thermophys Heat Transf 2009;23(3):543-50.

[5] Lander H, Nixon AC. Endothermic Fuels for Hypersonic Vehicles. J Aircr $1971 ; 8(4): 200-7$.

[6] Dang G, Zhong F, Zhang Y, Zhang X. Numerical study of heat transfer deterioration of turbulent supercritical kerosene flow in heated circular tube. Int J Heat Mass Transf 2015;5:1003-11.

[7] Bazargan M, Mohseni M. Algebraic zero-equation versus complex two-equation turbulence modeling in supercritical fluid flows. Comput Fluids 2012;60:49-57.

[8] AChouai SL, Richon D. Modeling of thermodynamic properties using neural networks application to refrigerants. Fluid Phase Equilib 2002;199:53-62.

[9] Yang V. Modeling of supercritical vaporization, mixing, and combustion processes in liquid-fueled propulsion system. Proc Combustion Inst 2000;28(2000):925-42.

[10] Azizi S, Ahmadloo E. Prediction of heat transfer coefficient during condensation of R134a in inclined tubes using artificial neural network. Appl Therm Eng 2016;106:203-10.

[11] Pini M, Spinelli A, Persico G, Rebay S. Consistent look-up table interpolation method for real-gas flow simulations. Comput Fluids 2015;107:78-188.

[12] Yao W. Kerosene-fueled supersonic combustion modelings based on skeletal mechanisms. Acta Mechanica Sinica 2019:1-23.

[13] Li X, Zhong F, Fan X, Huai X, Cai J. Study of turbulent heat transfer of aviation kerosene flows in a curved pipe at supercritical pressure. Appl Therm Eng 2010;30(13):1845-51

[14] Zhong F-Q, Fan X-J, Yu G, Li J-G, Sung C-J. Performance of supersonic model combustors with staged injections of supercritical aviation kerosene. Acta Mechanica Sinica 2010;26(5):661-8.

[15] Zhu J, Tao K, Tao Z, Qiu L. Heat transfer degradation of buoyancy involved convective RP-3 hydrocarbon fuel in vertical tubes with various diameters under supercritical pressure. Appl Therm Eng 2019;163:114392.

[16] Lian, C., Solution limited time stepping method and numerical simulation of single-element rocket enging combustor, PHD, Vol. West Lafayette,Indiana, No. Purdue University. (2009).

[17] Fan Xue-jun YG. Analysis of thermophysical properties of daqing RP-3 aviation kerosene. J Propulsion Technol 2006;27(2):187-92.

[18] Di Cheng XWaXF. Thermophysical Surrogate modelling of RP-3 aviation kerosene. The Seventh National Fluid Mechanics Conference, GuiLin, China, December; 2012.

[19] Zhong FQ, Fan XJ, Wang J, Yu G, Li JG. Characteristics of compressible flow of supercritical kerosene. Acta Mechanica Sinica 2012;28(1):8-13.

[20] Peng DYaR DB. A new two-constant equation of state. Ind Eng Chem Fundam $1976 ; 15: 59-64$.

[21] Redlich O, Kwong JNS. On the thermodynamics of solutions. Chem Rev 1949;44(233).

[22] Gary D, Fisher TWL Jr. Corresponding states principle using shape factors. Ind Eng Chem Fundam 1970;9(4).

[23] Queimada AJ, Stenby EH, Marrucho IM, Coutinho JAP. A new corresponding states model for the estimation of thermophysical properties of long chain N-Alkanes. Fluid Phase Equilib 2003;212(1-2):303-14.

[24] Zhu J, Tao Z, Deng H, Wang K, Yu X. Numerical investigation of heat transfer characteristics and flow resistance of kerosene RP-3 under supercritical pressure. Int J Heat Mass Transf 2015;91:330-41.

[25] Leach JW, Chappelear PS, Leland TW. Use of molecular shape factors in vapor-liquid equilibrium calculations with the corresponding states principle. AIChE J 2004;14(4):568-76.

[26] Oefelein JC. Thermophysical characteristics of shear-coaxial LOX-H2 flames at supercritical pressure. Proc Combust Inst 2005;30:2929-37.

[27] Hempert F, Boblest S, Ertl T, Sadlo F, Offenhäuser P, Glass CW, et al. Simulation of real gas effects in supersonic methane jets using a tabulated equation of state with a discontinuous Galerkin spectral element method. Comput Fluids 2017; 145:167-79.

[28] Kocabaş F, Ünal S, Ünal B. A neural network approach for prediction of critical submergence of an intake in still water and open channel flow for permeable and impermeable bottom. Comput Fluids 2008;37:1040-6.

[29] Sen B, Menon S. Representation of chemical kinetics by artificial neural networks for large eddy simulations. AIAA/ASME/SAE/ASEE joint propulsion conference \& exhibit/43rd; 2007.

[30] Zhou Z, He G, Wang S, Jin G. Subgrid-scale model for large-eddy simulation of isotropic turbulent flows using an artificial neural network. Comput Fluids 2019;195:104319

[31] Kalogirou SA. Applications of artificial neural-networks for energy systems. Appl Energy 2000;67:17-35.

[32] Şencan A, Kalogirou SA. A new approach using artificial neural networks for determination of the thermodynamic properties of fluid couples. Energy Convers Manage 2005;46(15-16):2405-18.
[33] Li C, Yu C. Performance evaluation of public non-profit hospitals using a Bp artificial neural network: the case of Hubei Province in China. Int J Environ Res Public Health 2013;10(8):3619-33.

[34] Lim HS, Kang YT. Estimation of finish cooling temperature by artificial neural networks of backpropagation during accelerated control cooling process. Int J Heat Mass Transf 2018;126:579-88.

[35] Mora R JE, Pérez T C, González N FF, Ocampo D JDD. Thermodynamic properties of refrigerants using artificial neural networks. Int J Refriger 2014;46:9-16.

[36] Longo GA, Zilio C, Ceseracciu E, Reggiani M. Application of Artificial Neural Network (Ann) for the prediction of thermal conductivity of oxide-water nanofluids. Nano Energy 2012;1(2):290-6.

[37] Zhao N, Li Z. Experiment and artificial neural network prediction of thermal conductivity and viscosity for alumina-water nanofluids. Materials (Basel) 2017;10(5).

[38] Choi Y, Chen JY. Fast prediction of start-of-combustion in hcci with combined artificial neural networks and ignition delay model. Proc Combust Inst 2005;30(2):2711-18.

[39] Sablani SS, Kacimov A, Perret J, Mujumdar AS, Campo A. Non-iterative estimation of heat transfer coefficients using artificial neural network models. Int J Heat Mass Transf 2005;48(3-4):665-79.

[40] Rahman,A A, Zhang X. Prediction of oscillatory heat transfer coefficient for a thermoacoustic heat exchanger through artificial neural network technique. Int J Heat Mass Transf 2018;124:1088-96.

[41] Milan PJ, Li Y, Wang X, Yang S, Sun W, Yang V. Time-efficient methods for real fluid property evaluation in numerical simulation of chemically reacting flows. In: 11th US national combustion meeting; 2019. p. 1-10. 71TF-0396.

42] Milan PJ, Wang X, Hickey J-P, Li Y, Yang V. Accelerating numerical simulations of supercritical fluid flows using deep neural networks. In: AIAA Scitech 2020 forum, 1157; 2020. p. 1-2.

[43] Owoyele O, Kundu P, Ameen MM, Echekki T, Som S. Application of deep artificial neural networks to multi-dimensional flamelet libraries and spray flames. Int J Engine Res 2019;21(1):151-68.

[44] Ihme M. Construction of optimal artificial neural network architectures for application to chemical systems: comparison of generalized pattern search method and evolutionary algorithm. Artif Neural Netw 2019;20(2):125-50.

[45] Liu Z, Liang J, Pan Y. Construction of thermodynamic properties look-up table with block-structured adaptive mesh refinement method. J Thermophys Heat Transf 2014:28(1):50-8.

[46] Yang S, Li Y, Wang X, Unnikrishnan U, Yang V, Sun W. Comparison of tabulation and correlated dynamic evaluation of real fluid properties for supercritical mixing. 53rd AIAA/SAE/ASEE joint propulsion conference; 2017.

[47] Rumelhart DEH, Geoffrey E, Williams, Ronald J. Learning Representations by Back-Propagating Errors. Nature 1986;323:533-6.

[48] Schmidhuber J. Deep learning in neural networks: an overview. Neural Netw 2015;61:85-117.

[49] Mizutani Eiji. On derivation of Mlp backpropagation from the Kelley-Bryson optimal-control gradient formula and its application. In: Proceedings of the IEEE International joint conference on neural networks; 2000.

[50] Li Y, Fu Y, Li H, Zhang S-W. The improved training algorithm of back propagation neural network with self-adaptive learning rate. In: 2009 international conference on computational intelligence and natural computing; 2009. p. 73-6.

[51] Fiesler E. Neural networks with adaptive learning rate and momentum terms; 1995. IDIAP Technical report95-04.

[52] Nishiumi H, Arai T. Generalization of the binary interaction parameter of the peng-robinson equation of state. Fluid Phase Equilibra 1988;42:43-62.

[53] Polyakov VB, Cole JHDR, Chialvo AA. Novel corresponding-states principle approach for the equation of state of isotopologues: $\mathrm{H}_{2}{ }^{18} \mathrm{O}$ as an example. J Phys Chem 2007;111:393-401.

[54] Meng Yang. High-speed pulse schlieren technology and its role in the flow field display of supersonic combustor, BeiJing. University of Chinese Academy of Science; 2012

[55] Yao W, Lu Y, Li X, Wang J, Fan X. Improved delayed detached eddy simulation of a high-Ma regenerative-cooled scramjet combustor based on skeletal kerosene mechanism. AIAA 2016.

[56] Weller HG, Tabor G, Jasak H, Fureby C. A tensorial approach to CFD using object oriented techniques. Comput Phys 1997;12(6):620-31.

[57] Yao W, Wang J, Lu Y, Li X, Fan X. Full-scale detached eddy simulation of kerosene fueled scramjet combustor based on skeletal mechanism. 20th AIAA international space planes and hypersonic systems and technologies conference, Glasgow, Scotland; 2015. 6-9 July.

[58] Li X, Yao W, Fan X. Large-eddy simulation of time evolution and instability of highly underexpanded sonic jets. AIAA J 2016;54(10):3191-211.

[59] Li X, Zhou R, Yao W, Fan X. Flow characteristic of highly underexpanded jets from various nozzle geometries. Appl Therm Eng 2017;125:240-53.

[60] Won-Wook K, Suresh M. A new dynamic one-equation subgrid-scale model for large eddy simulations. 33rd aerospace sciences meeting and exhibit, Reno, NV, January 9-12; 1995.

[61] Kurganov A, Tadmor E. New high-resolution central schemes for nonlinear conservation laws and convection-diffusion equations. J Comput Phys 2000:160(1):241-82.

[62] Vuorinen V, Larmi M, Schlatter P, Fuchs L, Boersma BJ. A low-dissipative, scale-selective discretization scheme for the Navier-Stokes equations. Comput Fluids 2012;70:195-205 